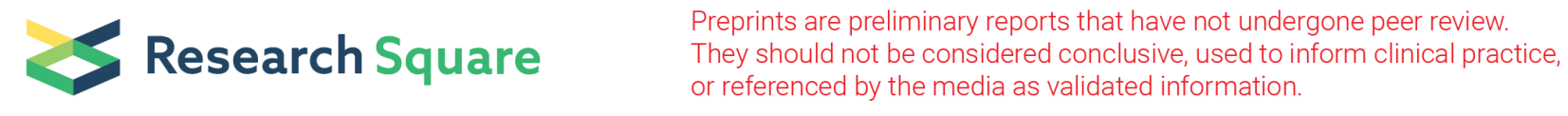

\title{
Coexistence of Mother Overweight and Stunting Children Within the Same Household in West Africa : Associated Factors
}

Stanislav Seydou TRAORE

Zhengzhou University https://orcid.org/0000-0001-8950-4827

Adwoa Nyantakyiwaa Amoah

Zhengzhou University

Han Zhang

Zhengzhou University

Youchun $\mathrm{Hu}$

Zhengzhou University

Quanjun Lyu ( $\sim$ lqjinutr@zzu.edu.cn )

science Avenue, College of Public Health and the First affiliated Hospital of Zhengzhou University, Zhengzhou, China,

Research article

Keywords: Overweight, Stunting, Double burden of malnutrition, West Africa

Posted Date: October 29th, 2020

DOI: https://doi.org/10.21203/rs.3.rs-97999/v1

License: (c) (i) This work is licensed under a Creative Commons Attribution 4.0 International License. Read Full License 


\section{Abstract}

\section{Background}

The double burden of malnutrition is the new reality in many low- and middle-income countries. Updating and meeting the challenge of double burdens on vulnerable people is essential to ensure healthy growth. This study is to explore the individual or household factors that may be associated with coexistence of mother overweight and stunting children within the same household (MOSC) in the West African countries. Besides, we sought to examine its prevalence using data from national surveys.

\section{Methods:}

We used nationally representative data from the Demographic and Health Survey of 11 West African countries. Data years range from 2012 to 2014 . We used logistic regression to explore the association between the occurrence of coexistence of mother overweight and stunting children within the same household and individual or household factors. Logistic regression was performed on the pooled data to allow comparison between countries ranked by gross national income.

\section{Results:}

The Prevalence of MOSC was higher in Benin 11.52\% (95\% Cl, 10.61-12.43), the lowest proportion was in Togo and Guinea [4.83\% (95\% Cl, 3.88-5.77), 4.48\% $(95 \% \mathrm{Cl}, 3.57-5.40)$, respectively]. Women who did not receive antenatal cares were associated with higher odds of MOSC occurrence compared to those who received more than 4 antenatal cares in all countries except Gambia, this association was significant in 7 countries among which the MOSC in Ghana was four times more likely to occur in women who did not receive antenatal care compared to more than 4 antenatal cares [odds ratio (95\% Cl): 4.936 (2.7019.019)]. Children over 24 months of age were significantly associated with higher odds of MOSC occurrence compared to under 24 months in all countries except Sierra Leone [odds ratio $(95 \% \mathrm{Cl}): 1.215(0.867-1.703)]$.

\section{Conclusions:}

In this study, none of the individual and household factors were strongly associated in the same way with MOSC occurrence in West African countries, they varied considerably between national databases and independent of national income per capita. However, Children's age and antenatal cares were the two covariates strongly associated with MOSC occurrence in most countries.

\section{Background}

The double burden of malnutrition is the new reality in many low- and middle-income countries (LMICs), and serious forms are in Sub-Saharan Africa and some parts of Asia [1, 2]. The Global Nutrition Report reported multiple forms of malnutrition's burden among Children under 5 years of age and mother of reproductive age in several countries especially in West Africa [2,3]. Understanding in a specific way who and where are most affected by the prevalence of these forms of malnutrition is a need to contribute to the implementation of political decisions and the direction of new research.

For many years, many countries have struggled to reduce the prevalence of undernutrition with slight progress, now they must cope with the recent increase in overweight due to the phenomenon of food transition among the population [4, 5]. In 2018, more than 2.28 billion people around the world are estimated to be overweight and 150.8 million children under the age of five are stunted $[2,6]$.

In Africa, the prevalence of stunting children decreased from $38 \%$ in 2000 to $30 \%$ in 2018 , this is still above $25 \%$ which is the average of stunting in LMICs. And the mother overweight has increased from $31.7 \%$ in 2000 to $41.1 \%$ in 2016 [7]. This rise in overweight is mainly due to the rapid change in eating habits, but also the socio-cultural influence in which obesity is considered a beauty for mother and a fulfilling life for men [8]. The double burden of malnutrition is the coexistence of undernutrition and overweight in the same population, household or individual [1, 2], And studies have indicated that it is most prevalent in LMIC where undernutrition is still a public health problem [4].

In this study we have focused on the coexistence of mother overweight and stunting children in the same household (MOSC). We preferred stunting over underweight or wasting as an indicator of child undernutrition because stunting has a negative impact on linear growth in infancy, impaired cognitive development, it is associated with the risk of morbidity, mortality, and noncommunicable diseases in later life. On the other hand, underweight is a nonspecific indicator of overall malnutrition, while wasting is an emergency because of its association with a high risk of death. $[9,10]$

Studies have already been carried out on the MOSC in LMICs, the result of which found a general prevalence of less than $10 \%$ and concluded that it could increase rapidly in the future. Author reported an association between the prevalence of MOSC and economic development in Africa [11, 12]. A recent study of pooled data from 15 countries in Sub-Saharan Africa to assess the association of deforestation with nutritional status across all countries [13], the authors found that the prevalence of mother overweight and stunting children in the same household was $6.9 \%$, factors strongly associated with it included the mother's age, living in urban, household size, improved sanitation facility, child's age, child's gender, forest cover in 2000, and distance to the nearest road [13].

Updating and meeting the challenge of double burdens on vulnerable people is essential to ensure healthy growth and development of the population and to avoid long-term consequences for future generations [14, 15]. In this study, we wanted to find out if the association of individual and household factors on the coexistence of overweight mother and stunted children (MOSC) in the same household are the same for each of the West African countries.

The objective of this study is to explore the individual or household factors that may be associated with MOSC in the West African countries. Besides, we sought to examine the prevalence of MOSC using data from national surveys. 


\section{Methods}

Analyses were performed with available nationally representative data from the West African countries' Demographic and Health Survey (DHS). Out of the 17 countries of West Africa, DHS data of 11 countries were available and contained mother and their children body measurements. The years of the data range from 2012 to 2014. The DHS Program is designed to provide monitoring data of the population particularly children and women in developing countries and evaluate health and nutritional situation. Data for the population sample are collected with the collaboration of the governments of the countries. It is a stratified two-stage cluster design and based on the selection of households to provide an adequate representation of urban and rural areas and geographical region. Data sets are available from the DHS website (https://dhsprogram.com/) [16]. The ICF provided technical assistance through the program, whose objective is to provide support to countries for carrying out demographic and health surveys [17]. Our study evaluated the association of interest in a representative sample of women of childbearing 15-49 years of age and their children under 5 years of age. We have chosen the 'Kids Recode' files which contain all the information relating to children and mother. We excluded all mother with non-plausible anthropometric data, non-pregnant, recently postpartum, having a height under $135 \mathrm{~m}$ and BMI less than $12 \mathrm{~kg} / \mathrm{m}^{2}$ or higher than $60 \mathrm{~kg} / \mathrm{m}^{2}$; and children with non-plausible anthropometric data and height/age less than 6 or higher than $6 z$-scores. If a mother had more than one child, we selected one of the children who was stunted or the last born if none of them was stunted.

Nutritional status was assessed following the World Health Organization reference standards (maternal body mass index (BMI) and height for age z-scores (HAZ) for children). Stunting children was defined as a HAZ $\leq-2$ SD of the WHO/NCHS/CDC new reference standards (WHO, 2006). Mother overweight was defined as $\mathrm{BMI} \geq 25.0 \mathrm{~kg} / \mathrm{m}^{2}$. The MOSC (defined as the coexistence of mother overweight and stunting children in the same household) was determined by the presence in the household of overweight in a mother and stunting in at least one of her children. All outcomes variables were coded as a binary variable. We included several Independent variables. We selected these covariates based on previous evidence of the determinants of nutritional status [13, 18]. The mother-level covariates included place of residence (categorized as Urban and Rural), Wealth index (Poorest, Poorer, Middle, Richer, Richest), age group (categorized as 15-19, 20-29, 30-39, 40-49), Education level (categorized as None, Primary, $\geq$ Secondary), Access to information (categorized as No and Yes), Source of water (categorized as Improved and Unimproved), Sanitation facility (categorized as Improved and Unimproved), the number of members in households (categorized as $\leq 5$ members, $>5$ members), Antenatal Care (categorized as None, 3 times and less, more than 4 times). The child-level covariates included child age ( categorized as $\leq 24$ months and $>24$ months), child gender ( categorized as Male and Female), Child small birth Weight ( categorized as Small, normal or Large and Missing), Child diarrhea during the last 2 weeks (categorized as No and Yes) and Month of birth (categorized as January, February, March, April, May, June, July, August, September, October, November and December).

\section{Statistical analysis:}

This study aims to examine country-specific relationships between the independent variable and the MOSC occurrence and to conduct cross-country comparisons of the MOSC prevalence. Therefore, we present results as summary tables and make qualitative comparisons across studies. We calculated proportions for the outcome and independent variables, as well as for key characteristics. Multivariate logistic regressions were employed to estimate the association between prevalence of MOSC and the individual and household factors. Logistic regression was performed on the pooled data to allow comparison between countries ranked by gross national income. All analyses were adjusted for the weights and complex survey design of the DHS as recommended by the Guide to DHS Statistics DHS-7 [17]. The analyses were conducted using the Statistical Package for the Social Sciences (SPSS) software (Version 24.0) and Statistical Analysis Systems statistical (SAS) Studio University Edition. A 2-sided $P$ value of .05 was used to assess statistical significance.

\section{Results}

\section{Descriptive data}

Out of the 17 countries of West African countries, DHS data of 11 countries that were available and contain body measurements of mother and their children were analyzed. The years of survey were from 2012 to 2014. Datasets from demographic and health surveys by country used in the analysis, classified from lowest to highest in their Gross National Income (GNI) per capita (equivalent in US dollars) are shown in the table1.

Table 1: Country Demographic and Health Survey datasets used in the analysis. 


\begin{tabular}{|lllll|}
\hline Country & Year of Survey (DHS) & Rank & GNI_US\$ & Total \\
\hline GAMBIA & 2013 & 1 & $1,474.43$ & 1839 \\
\hline LIBERIA & 2013 & 2 & $2,302.90$ & 2074 \\
\hline SIERRA LEONE & 2013 & 3 & $3,902.99$ & 2827 \\
\hline TOGO & $2013-14$ & 4 & $4,414.57$ & 2065 \\
\hline GUINEA & 2012 & 5 & $7,987.54$ & 1980 \\
\hline NIGER & 2012 & 6 & $9,784.66$ & 2523 \\
\hline MALI & $2011-12$ & 7 & $11,669.65$ & 2516 \\
\hline BENIN & 2012 & 8 & $11,676.53$ & 4897 \\
\hline IVORY COAST & $2011-12$ & 9 & $30,442.68$ & 1988 \\
\hline GHANA & 2014 & 10 & $42,833.02$ & 1778 \\
\hline NIGERIA & 2013 & 11 & $438,453.56$ & 13915 \\
\hline
\end{tabular}

GNI = per capita Gross National Income; Source: World Bank 2015 Country Data and Statistics website, accessed July 2020.

DHS: Demographic and Health Survey.

Table2 presents the descriptive characteristics of the analytical sample of 11 countries based on Demographic and Health Surveys, the sample population in each country except Liberia was predominantly rural. Liberia, Gambia and Ghana samples had the lowest proportion of rural households (46\%, $52.8 \%$ and $52.7 \%$, respectively) and the highest proportion was in Niger and Mali ( $80.8 \%$ and $79.3 \%$, respectively). The proportion of household wealth index was predominantly that of the poor group in all countries regardless of the GNI per capita of countries (World Bank in 2019) except Niger and Benin in which the proportion of the rich group was higher $(40.9 \%, 41.6 \%$, respectively). The predominant mother's age group in each country was those between 20 to $39-y e a r$. The proportion of the mother who had secondary education or more was extremely low in Niger and Mali (4.9\%, $8.3 \%$ respectively), while in Ghana, this proportion was much larger (53\%). Over $60 \%$ of mother in each country had access to at least one source of information, the highest proportion was reported in Ghana and Gambia ( $90.2 \%$ and $90.1 \%$ respectively). More than $60 \%$ of the household in each country except Sierra Leone (56.\%) had access on improved water sources, while the access to improved sanitary facilities was low in each country, Sierra Leone and Niger had the lowest proportion of $8.2 \%$ and $9.4 \%$, respectively, and the highest of $37.5 \%$ was in the Gambia. More than half of households in all countries except Ghana (42.4\%) were predominantly made up of 5 or more members. The proportion of mother who received 4 or more antenatal cares was lower in Niger and Mali (27.9\% and $35.7 \%$, respectively) and the higher proportion was reported in Ghana and Liberia ( $83.2 \%$ and $71.2 \%$, respectively). The proportion of small birth weight was higher in Gambia and Ghana (50.9\% and 51.3\%, respectively), while the lowest proportion was in Benin and Niger (18.7\% and 19.5\%, respectively). The proportion of children who had Diarrhea in the last two weeks preceding the interview was higher in Liberia, Gambia and Ivory Coast (25.5\% 21.1\%, 21.5, respectively), while the lowest proportion was reported in Benin and Mali (7.9\% and 9.3\%, respectively).

Figure1 shows the prevalence of mother overweight, stunting children and MOSC by country ranked by their Gross National Income per capita (equivalent in US Dollars). The prevalence of overweight was higher in Ghana $40.31 \%$ (95\% Cl, 37.99-42.64), while the lowest proportion was in Sierra Leone 15.70\% (95\% $\mathrm{Cl}$, 14.37-17.03). The prevalence of stunting was higher in Niger $53.46 \%(95 \% \mathrm{Cl}, 51.53-55.39)$, while the lowest prevalence was reported in $\mathrm{Ghana} 21.3 \%(95 \% \mathrm{Cl}$, 19.36-23.24). The Prevalence of MOSC was higher in Benin 11.52\%(95\% Cl,10.61- 12.43), the lowest proportion was in Togo and Guinea [4.83\% (95\% $\mathrm{Cl}, 3.88-$ 5.77), 4.48\% (95\% Cl, 3.57-5.40), respectively].

To know if the different factors are associated with MOSC occurrence in countries, we compared the prevalence of MOSC between countries by different individual and household characters which is represented in the Table 3 . The results showed that the MOSC appeared to differ significantly when the countries were compared to the one with the highest gross income per capita, except for 3 categories of two covariates which are the mothers in the youngest group of age of 15-19 (P-value $=0.641)$ and the months of March and December (P-value: 0.172 and 0.255 , respectively).

\section{Association between individual/household factors and the MOSC}

Table 4 shows the result of logistic regression for the association of each factor with the MOSC (Mother overweight and stunting children in the same household), by country ranked by their GNI. Among the covariates, living in the urban area was associated with higher odds of MOSC occurrence in each country except Guinea and Togo, however, the urban area was significantly associated to MOSC occurrence in 4 countries which are Niger, Mali, Nigeria and Ivory Coast [odds ratio $(95 \% \mathrm{Cl}): 2.545$ (1.644-3.94), 1.906 (1.129-3.217), 1.248 (1.059-1.47) and 2.425 (1.188-4.948), respectively]. The household's wealth was strongly associated with the MOSC occurrence in Liberia, Sierra Leone, Togo, Benin and Nigeria. It was rich and middle wealth households that were more associated with higher odds of MOSC occurrence compared to poor households in each country except in Ivory Coast [odds ratio (95\% Cl): 0.546(0.269-1.108), 0.605(0.264-1.387), respectively]. Mother's age showed a significant association with MOSC occurrence in 5 countries which are Liberia, Niger, Mali, Benin and Nigeria. Mother with no or primary education were associated with higher odds of MOSC occurrence compared to secondary or higher education in all countries except in Togo, however, MOSC was more than three times more likely to be significantly associated to mother with no education level in Gambia [odds ratio $(95 \% \mathrm{Cl}): 3.737(1.833-7.618)]$, and more than two times in Ivory Coast [odds ratio $(95 \% \mathrm{Cl}): 2.911(1.152-7.356)$ ], while the primary education was 
significantly associated with higher odds of MOSC occurrence in three countries: Ivory Coast (with more than three times), Gambia (with more than four times) and Nigeria [odds ratio (95\% Cl): 3.259 (1.26-8.431), 4.473 (1.936-10.332), 1.252 (1.044-1.502), respectively]. Access to information was significantly associated with lower odds of MOSC occurrence in The Gambia [odds ratio (95\% Cl): $0.505(0.281-0.907)$ ], while in Ivory Coast and Nigeria, access to information was significantly associated with higher odds of MOSC occurrence (two times as many as households without access to information in Ivory Coast) [odds ratio $(95 \% \mathrm{Cl})$ : 2.335 (1.403-3.885), 1.208 (1.023-1.426), respectively]. Household access to the unimproved water source was significantly associated with lower odds of MOSC occurrence in Ghana [odds ratio $(95 \% \mathrm{Cl}): 0.191(0.061-0.597)$ ]. Household Access to the unimproved sanitation was significantly associated with higher odds of MOSC occurrence in Liberia [odds ratio ( $95 \% \mathrm{Cl}): 2.815(1.223-6.48)$ ], while that association was significant with lower odds of MOSC occurrence in Nigeria [odds ratio (95\% Cl): $0.855(0.746-0.98)$ ]. The household with more than 5 members was associated with higher odds of MOSC occurrence in all countries, however, it was significant in the three highest Gross National Income countries: Ivory Coast, Ghana and Nigeria [odds ratio $(95 \% \mathrm{Cl})$ : 1.66 (1.055-2.61), 1.873 (1.148-3.056), 1.248 (1.078-1.445), respectively]. Women who did not receive antenatal cares were associated with higher odds of MOSC occurrence compared to those who received more than 4 antenatal cares in all countries except Gambia, this association was significant in 7 countries among which the MOSC in Ghana was four times more likely to occur in women who did not receive antenatal care compared to more than 4 antenatal cares [odds ratio $(95 \% \mathrm{Cl}): 4.936$ (2.701-9.019)]. In Guinea and Togo, the statistical significance of the association between antenatal cares and MOSC occurrence was at the borderline ( $P$-value $=0.05$ and 0.057 , respectively), however, the lack of prenatal cares was significantly associated with higher odds of MOSC occurrence [odds ratio $(95 \% \mathrm{Cl}): 2.000$ (1.145-3.494) and 2.04 (1.122-3.711), respectively]. Female gender was strongly associated with higher odds of MOSC occurrence in Sierra Leone and Mali [odds ratio (95\% Cl): 1.42 (1.028-1.962), 1.466 (1.032-2.083), respectively], while it was marginally or non-significantly associated with lower odds of MOSC occurrence in other countries. Children over 24 months of age were significantly associated with higher odds of MOSC occurrence compared to under 24 months in all countries except Sierra Leone [odds ratio (95\%Cl): 1.215 (0.867-1.703)]. Small birth weight was significantly associated with lower odds of MOSC occurrence in Ivory Coast [odds ratio $(95 \% \mathrm{Cl})$ : $0.577(0.384-0.868)$ ]; while it was significantly associated with higher odds of MOSC occurrence in Niger [odds ratio $(95 \% \mathrm{Cl}): 1.498(1.054-2.131)$ ]. Diarrhea in the past two weeks among children was marginal or no associated with the MOSC occurrence in each country.

Children month of birth was significantly associated with MOSC occurrence in Benin, Ivory Coast, Ghana and Nigeria which have the highest GNI 2015 per capita.

Table 2: Descriptive characteristics of the analytical sample of 11 countries based on Demographic and Health Surveys, $2012-2014$. 


\begin{tabular}{|c|c|c|c|c|c|c|c|c|c|c|c|c|}
\hline & \multirow{2}{*}{$\begin{array}{l}\text { Place of } \\
\text { residence } \\
\text { Rural }\end{array}$} & \multicolumn{3}{|l|}{ wealth } & \multicolumn{4}{|c|}{ Mother age in years } & \multicolumn{3}{|c|}{ Mother education } & \multirow{2}{*}{$\begin{array}{l}\text { Access to } \\
\text { information } \\
\text { Yes }\end{array}$} \\
\hline & & Poor & Middle & Rich & $20-29$ & $30-39$ & $40-49$ & $15-19$ & None & Primary & Secondary & \\
\hline & N (\%) & N (\%) & N (\%) & N (\%) & $\mathrm{N}(\%)$ & $\mathrm{N}(\%)$ & N (\%) & $\mathrm{N}(\%)$ & N (\%) & $\mathrm{N}(\%)$ & N (\%) & $\mathrm{N}(\%)$ \\
\hline Gambia & $\begin{array}{l}1204 \\
(52.8)\end{array}$ & $951(43.5)$ & $341(17.2)$ & $\begin{array}{l}547 \\
(39.3)\end{array}$ & $\begin{array}{l}867 \\
(47.8)\end{array}$ & $\begin{array}{l}675 \\
(36.5)\end{array}$ & $\begin{array}{l}196 \\
(10.6)\end{array}$ & $\begin{array}{l}101 \\
(5.1)\end{array}$ & $\begin{array}{l}1139 \\
(58.2)\end{array}$ & $\begin{array}{l}261 \\
(13.6)\end{array}$ & $439(28.2)$ & $1613(90.1)$ \\
\hline Liberia & $\begin{array}{l}1368 \\
(46.2)\end{array}$ & $\begin{array}{l}1262 \\
(43.6)\end{array}$ & $406(19.6)$ & $\begin{array}{l}406 \\
(36.8)\end{array}$ & $\begin{array}{l}962 \\
(48.6)\end{array}$ & $\begin{array}{l}675 \\
(31.2)\end{array}$ & $\begin{array}{l}242 \\
(10.1)\end{array}$ & $\begin{array}{l}195 \\
(10.1)\end{array}$ & $962(39.8)$ & $\begin{array}{l}674 \\
(29)\end{array}$ & 438 (31.2) & $1380(72.5)$ \\
\hline Sierra L & $\begin{array}{l}1960 \\
(75.1)\end{array}$ & $\begin{array}{l}1201 \\
(44.6)\end{array}$ & $578(21.6)$ & $\begin{array}{l}1048 \\
(33.8)\end{array}$ & $\begin{array}{l}1217 \\
(43.5)\end{array}$ & $\begin{array}{l}1083 \\
(37.9)\end{array}$ & $\begin{array}{l}294 \\
(10.9)\end{array}$ & $\begin{array}{l}233 \\
(7.7)\end{array}$ & $\begin{array}{l}1904 \\
(67.7)\end{array}$ & $\begin{array}{l}388 \\
(14.3)\end{array}$ & $535(18)$ & $1730(60.2)$ \\
\hline Togo & $\begin{array}{l}1467 \\
(63.3)\end{array}$ & $\begin{array}{l}1032 \\
(40.4)\end{array}$ & $388(20)$ & $\begin{array}{l}645 \\
(39.5)\end{array}$ & $\begin{array}{l}868 \\
(42.9)\end{array}$ & $\begin{array}{l}809 \\
(39.3)\end{array}$ & $\begin{array}{l}284 \\
(12.8)\end{array}$ & $\begin{array}{l}104 \\
(5)\end{array}$ & $885(38.1)$ & $\begin{array}{l}739 \\
(38.2)\end{array}$ & $441(23.7)$ & $1350(68.2)$ \\
\hline Guinea & $\begin{array}{l}1408 \\
(73.6)\end{array}$ & $866(44.6)$ & $388(19.8)$ & $\begin{array}{l}726 \\
(35.7)\end{array}$ & $\begin{array}{l}851 \\
(43.1)\end{array}$ & $\begin{array}{l}676 \\
(33.9)\end{array}$ & $\begin{array}{l}236 \\
(11.6)\end{array}$ & $\begin{array}{l}217 \\
(11.3)\end{array}$ & $1535(77)$ & $\begin{array}{l}239 \\
(12.4)\end{array}$ & $206(10.5)$ & $1336(67.1)$ \\
\hline Niger & $\begin{array}{l}1908 \\
(85)\end{array}$ & $866(38.9)$ & $454(20.3)$ & $\begin{array}{l}1203 \\
(40.9)\end{array}$ & $\begin{array}{l}1159 \\
(45.5)\end{array}$ & $\begin{array}{l}921 \\
(36.5)\end{array}$ & $\begin{array}{l}286 \\
(11.4)\end{array}$ & $\begin{array}{l}157 \\
(6.7)\end{array}$ & $\begin{array}{l}2067 \\
(85.4)\end{array}$ & $\begin{array}{l}284 \\
(9.8)\end{array}$ & $172(4.9)$ & $1634(65)$ \\
\hline Mali & $\begin{array}{l}1851 \\
(79.3)\end{array}$ & $994(41.8)$ & $477(19.7)$ & $\begin{array}{l}1045 \\
(38.5)\end{array}$ & $\begin{array}{l}1155 \\
(45.5)\end{array}$ & $\begin{array}{l}908 \\
(35.9)\end{array}$ & $\begin{array}{l}270 \\
(11)\end{array}$ & $\begin{array}{l}183 \\
(7.6)\end{array}$ & $\begin{array}{l}2038 \\
(82.6)\end{array}$ & $\begin{array}{l}238 \\
(9.1)\end{array}$ & $240(8.3)$ & $1832(71.8)$ \\
\hline Benin & $\begin{array}{l}3042 \\
(59.5)\end{array}$ & $\begin{array}{l}2037 \\
(38.7)\end{array}$ & $\begin{array}{l}1047 \\
(19.7)\end{array}$ & $\begin{array}{l}1813 \\
(41.6)\end{array}$ & $\begin{array}{l}2291 \\
(47.2)\end{array}$ & $\begin{array}{l}1929 \\
(39.5)\end{array}$ & $\begin{array}{l}482 \\
(9.4)\end{array}$ & $\begin{array}{l}195 \\
(3.8)\end{array}$ & $\begin{array}{l}3438 \\
(67.5)\end{array}$ & $\begin{array}{l}856 \\
(18.3)\end{array}$ & $603(14.2)$ & $3350(70.4)$ \\
\hline Ivory C & $\begin{array}{l}1290 \\
(61.8)\end{array}$ & $966(46.1)$ & $390(19.4)$ & $\begin{array}{l}632 \\
(34.5)\end{array}$ & $\begin{array}{l}956 \\
(48.5)\end{array}$ & $\begin{array}{l}666 \\
(32.9)\end{array}$ & $\begin{array}{l}202 \\
(9.7)\end{array}$ & $\begin{array}{l}164 \\
(9)\end{array}$ & $\begin{array}{l}1295 \\
(63.2)\end{array}$ & $\begin{array}{l}489 \\
(26.2)\end{array}$ & $204(10.6)$ & $1250(66.1)$ \\
\hline Ghana & $\begin{array}{l}1034 \\
(52.7)\end{array}$ & $936(41.9)$ & $338(20)$ & $\begin{array}{l}504 \\
(38.1)\end{array}$ & $\begin{array}{l}708 \\
(39.5)\end{array}$ & $\begin{array}{l}747 \\
(43.4)\end{array}$ & $\begin{array}{l}251 \\
(12.9)\end{array}$ & $\begin{array}{l}72 \\
(4.2)\end{array}$ & $606(27.1)$ & $\begin{array}{l}367 \\
(19.9)\end{array}$ & $805(53)$ & $1565(90.2)$ \\
\hline \multirow[t]{3}{*}{ Nigeria } & $\begin{array}{l}9045 \\
(62.8)\end{array}$ & $5896(43)$ & $\begin{array}{l}2852 \\
(19.4)\end{array}$ & $\begin{array}{l}5167 \\
(37.6)\end{array}$ & $\begin{array}{l}6097 \\
(44.3)\end{array}$ & $\begin{array}{l}5270 \\
(37.7)\end{array}$ & $\begin{array}{l}1762 \\
(12.4)\end{array}$ & $\begin{array}{l}786 \\
(5.7)\end{array}$ & $\begin{array}{l}6022 \\
(45.6)\end{array}$ & $\begin{array}{l}2945 \\
(20.1)\end{array}$ & $\begin{array}{l}4948 \\
(34.3)\end{array}$ & $9370(68.5)$ \\
\hline & $\begin{array}{l}\text { Source of } \\
\text { water }\end{array}$ & $\begin{array}{l}\text { Sanitation } \\
\text { Facility }\end{array}$ & $\begin{array}{l}\text { Household } \\
\text { members }\end{array}$ & \multicolumn{3}{|c|}{ Antenatal cares } & $\begin{array}{l}\text { Child } \\
\text { age }\end{array}$ & & $\begin{array}{l}\text { Child } \\
\text { gender }\end{array}$ & \multicolumn{2}{|c|}{$\begin{array}{l}\text { Small birth } \\
\text { size }\end{array}$} & $\begin{array}{l}\text { Child } \\
\text { diarrhea }\end{array}$ \\
\hline & Improved & Improved & $>5$ & None & $\begin{array}{l}<3 \\
\text { Times }\end{array}$ & $\begin{array}{l}>4 \\
\text { Times }\end{array}$ & $\leq 24$ & $>24$ & Male & No & Yes & Yes \\
\hline Gambia & $\begin{array}{l}1597 \\
(88)\end{array}$ & $601(37.5)$ & $\begin{array}{l}1614 \\
(87.8)\end{array}$ & $\begin{array}{l}285 \\
(14.6)\end{array}$ & $\begin{array}{l}300 \\
(16)\end{array}$ & $\begin{array}{l}1254 \\
(69.5)\end{array}$ & $\begin{array}{l}1106 \\
(59.5)\end{array}$ & $\begin{array}{l}733 \\
(40.5)\end{array}$ & $974(53.2)$ & $\begin{array}{l}902 \\
(49.1)\end{array}$ & 937 (50.9) & $403(21.1)$ \\
\hline Liberia & $\begin{array}{l}1347 \\
(71)\end{array}$ & $174(12)$ & $\begin{array}{l}1251 \\
(58.5)\end{array}$ & $\begin{array}{l}372 \\
(15.3)\end{array}$ & $\begin{array}{l}348 \\
(13.5)\end{array}$ & $\begin{array}{l}1354 \\
(71.2)\end{array}$ & $\begin{array}{l}1134 \\
(54.6)\end{array}$ & $\begin{array}{l}940 \\
(45.4)\end{array}$ & $\begin{array}{l}1093 \\
(54.6)\end{array}$ & $\begin{array}{l}1099 \\
(52.4)\end{array}$ & $975(47.6)$ & $566(25.5)$ \\
\hline Sierra L & $\begin{array}{l}1609 \\
(56.5)\end{array}$ & $250(8.2)$ & $1943(67)$ & $\begin{array}{l}712 \\
(26.5)\end{array}$ & $\begin{array}{l}230 \\
(8.4)\end{array}$ & $\begin{array}{l}1885 \\
(65.1)\end{array}$ & $\begin{array}{l}1425 \\
(50.6)\end{array}$ & $\begin{array}{l}1402 \\
(49.4)\end{array}$ & $\begin{array}{l}1366 \\
(47.7)\end{array}$ & $\begin{array}{l}1543 \\
(55.1)\end{array}$ & $\begin{array}{l}1284 \\
(44.9)\end{array}$ & $358(12.4)$ \\
\hline Togo & $\begin{array}{l}1251 \\
(61.9)\end{array}$ & $187(11.2)$ & $\begin{array}{l}1247 \\
(55.4)\end{array}$ & $\begin{array}{l}302 \\
(14)\end{array}$ & $\begin{array}{l}685 \\
(32.2)\end{array}$ & $\begin{array}{l}1078 \\
(53.9)\end{array}$ & $\begin{array}{l}1088 \\
(53.8)\end{array}$ & $\begin{array}{l}977 \\
(46.2)\end{array}$ & $\begin{array}{l}1052 \\
(51.4)\end{array}$ & $\begin{array}{l}1350 \\
(64.8)\end{array}$ & 715 (35.2) & $367(17.4)$ \\
\hline Guinea & $\begin{array}{l}1417 \\
(72.7)\end{array}$ & $371(17.1)$ & $\begin{array}{l}1457 \\
(74.2)\end{array}$ & $\begin{array}{l}445 \\
(22.4)\end{array}$ & $\begin{array}{l}532 \\
(27.4)\end{array}$ & $\begin{array}{l}1003 \\
(50.3)\end{array}$ & $\begin{array}{l}1120 \\
(56.9)\end{array}$ & $\begin{array}{l}860 \\
(43.1)\end{array}$ & $\begin{array}{l}1067 \\
(53.9)\end{array}$ & $\begin{array}{l}1058 \\
(51.6)\end{array}$ & $922(48.4)$ & 387 (19.5) \\
\hline Niger & $\begin{array}{l}1714 \\
(66.1)\end{array}$ & 341 (9.4) & $\begin{array}{l}1699 \\
(66.4)\end{array}$ & $\begin{array}{l}776 \\
(31.1)\end{array}$ & $\begin{array}{l}1023 \\
(41)\end{array}$ & $\begin{array}{l}724 \\
(27.9)\end{array}$ & $\begin{array}{l}1388 \\
(56.1)\end{array}$ & $\begin{array}{l}1135 \\
(43.9)\end{array}$ & $\begin{array}{l}1283 \\
(50.6)\end{array}$ & $\begin{array}{l}1990 \\
(80.5)\end{array}$ & 533 (19.5) & $452(19.1)$ \\
\hline Mali & $\begin{array}{l}1639 \\
(64)\end{array}$ & 559 (20.9) & $1642(66)$ & $\begin{array}{l}934 \\
(37.6)\end{array}$ & $\begin{array}{l}667 \\
(26.7)\end{array}$ & $\begin{array}{l}915 \\
(35.7)\end{array}$ & $\begin{array}{l}1228 \\
(49)\end{array}$ & $\begin{array}{l}1288 \\
(51)\end{array}$ & $\begin{array}{l}1274 \\
(51.2)\end{array}$ & $\begin{array}{l}1396 \\
(55.7)\end{array}$ & $\begin{array}{l}1120 \\
(44.3)\end{array}$ & $244(9.3)$ \\
\hline Benin & $\begin{array}{l}3800 \\
(78.5)\end{array}$ & $517(12.3)$ & $\begin{array}{l}2851 \\
(56.9)\end{array}$ & $\begin{array}{l}1335 \\
(27)\end{array}$ & $\begin{array}{l}1016 \\
(19.9)\end{array}$ & $\begin{array}{l}2546 \\
(53.1)\end{array}$ & $\begin{array}{l}2517 \\
(51.7)\end{array}$ & $\begin{array}{l}2380 \\
(48.3)\end{array}$ & $\begin{array}{l}2472 \\
(50.6)\end{array}$ & $\begin{array}{l}3995 \\
(81.3)\end{array}$ & 902 (18.7) & $390(7.9)$ \\
\hline Ivory C & $\begin{array}{l}1511 \\
(74.7)\end{array}$ & 338 (17.7) & $\begin{array}{l}1322 \\
(63.2)\end{array}$ & $\begin{array}{l}356 \\
(17)\end{array}$ & $\begin{array}{l}839 \\
(43)\end{array}$ & $\begin{array}{l}793 \\
(40)\end{array}$ & $\begin{array}{l}1148 \\
(59.2)\end{array}$ & $\begin{array}{l}840 \\
(40.8)\end{array}$ & 958 (47.5) & $\begin{array}{l}999 \\
(51.3)\end{array}$ & 989 (48.7) & 415 (21.5) \\
\hline Ghana & $\begin{array}{l}1493 \\
(85.5)\end{array}$ & $160(10)$ & $803(42.4)$ & $\begin{array}{l}167 \\
(9.3)\end{array}$ & $\begin{array}{l}155 \\
(7.5)\end{array}$ & $\begin{array}{l}1456 \\
(83.2)\end{array}$ & $\begin{array}{l}973 \\
(54.7)\end{array}$ & $\begin{array}{l}805 \\
(45.3)\end{array}$ & $929(53.6)$ & $\begin{array}{l}889 \\
(48.8)\end{array}$ & 889 (51.2) & 241 (12.6) \\
\hline \multirow[t]{3}{*}{ Nigeria } & $\begin{array}{l}8334 \\
(61.6)\end{array}$ & $\begin{array}{l}4297 \\
(30.8)\end{array}$ & $\begin{array}{l}8361 \\
(59.5)\end{array}$ & $\begin{array}{l}6245 \\
(46)\end{array}$ & $\begin{array}{l}1399 \\
(9.8)\end{array}$ & $\begin{array}{l}6271 \\
(44.2)\end{array}$ & $\begin{array}{l}7968 \\
(56.8)\end{array}$ & $\begin{array}{l}5947 \\
(43.2)\end{array}$ & $6988(50)$ & $\begin{array}{l}7707 \\
(55.9)\end{array}$ & $\begin{array}{l}6208 \\
(44.1)\end{array}$ & $1637(11.8)$ \\
\hline & \multicolumn{12}{|c|}{ Month of birth } \\
\hline & January & February & Mars & April & May & June & July & August & September & October & November & December \\
\hline Gambia & $\begin{array}{l}188 \\
(10.9)\end{array}$ & $146(8.6)$ & $127(6.9)$ & $\begin{array}{l}129 \\
(6.6)\end{array}$ & $\begin{array}{l}138 \\
(6.5)\end{array}$ & $\begin{array}{l}122 \\
(6.6)\end{array}$ & $\begin{array}{l}111 \\
(5.9)\end{array}$ & $\begin{array}{l}175 \\
(8.5)\end{array}$ & $178(9.4)$ & $\begin{array}{l}177 \\
(9.5)\end{array}$ & $183(9.5)$ & $165(11.2)$ \\
\hline
\end{tabular}




\begin{tabular}{|c|c|c|c|c|c|c|c|c|c|c|c|c|}
\hline Liberia & $183(8.7)$ & 135 (7.4) & $172(7.6)$ & $\begin{array}{l}180 \\
(8.3)\end{array}$ & $\begin{array}{l}199 \\
(9.9)\end{array}$ & $\begin{array}{l}194 \\
(9.1)\end{array}$ & $\begin{array}{l}180 \\
(9.2)\end{array}$ & $\begin{array}{l}154 \\
(7.2)\end{array}$ & $171(8.2)$ & $\begin{array}{l}152 \\
(7.5)\end{array}$ & 169 (8.7) & 185 (8.1) \\
\hline Sierra L & $253(8.9)$ & 244 (8) & $272(9.7)$ & $\begin{array}{l}329 \\
(11.7)\end{array}$ & $\begin{array}{l}296 \\
(10.3)\end{array}$ & $\begin{array}{l}278 \\
(9.8)\end{array}$ & $\begin{array}{l}234 \\
(8.8)\end{array}$ & $\begin{array}{l}199 \\
(7)\end{array}$ & 175 (6.2) & $\begin{array}{l}180 \\
(6.4)\end{array}$ & $173(6.2)$ & $194(7)$ \\
\hline Togo & 134 (6.6) & $141(6.6)$ & 179 (8.4) & $\begin{array}{l}190 \\
(9.3)\end{array}$ & $\begin{array}{l}225 \\
(11.4)\end{array}$ & $\begin{array}{l}173 \\
(8.5)\end{array}$ & $\begin{array}{l}157 \\
(7.5)\end{array}$ & $\begin{array}{l}175 \\
(8.5)\end{array}$ & 199 (9.8) & $\begin{array}{l}190 \\
(8.9)\end{array}$ & 157 (7.6) & $145(6.9)$ \\
\hline Guinea & 185 (8.9) & 204 (10.9) & $214(10.5)$ & $\begin{array}{l}212 \\
(10)\end{array}$ & $\begin{array}{l}217 \\
(10.6)\end{array}$ & $\begin{array}{l}200 \\
(10.6)\end{array}$ & $\begin{array}{l}153 \\
(8)\end{array}$ & $\begin{array}{l}126 \\
(6.3)\end{array}$ & $126(7.1)$ & $96(4.2)$ & $121(6.4)$ & $126(6.4)$ \\
\hline Niger & $\begin{array}{l}285 \\
(11.4)\end{array}$ & $274(11.1)$ & $277(10.8)$ & $\begin{array}{l}233 \\
(9)\end{array}$ & $\begin{array}{l}235 \\
(8.9)\end{array}$ & $\begin{array}{l}173 \\
(7.3)\end{array}$ & $\begin{array}{l}160 \\
(6.2)\end{array}$ & $\begin{array}{l}215 \\
(8.1)\end{array}$ & 199 (7.9) & 177 (7) & $137(5.6)$ & $158(6.8)$ \\
\hline Mali & 170 (7.1) & $183(7.1)$ & $227(9.1)$ & $\begin{array}{l}240 \\
(9)\end{array}$ & $\begin{array}{l}261 \\
(10.9)\end{array}$ & $\begin{array}{l}243 \\
(9.9)\end{array}$ & $\begin{array}{l}209 \\
(8.2)\end{array}$ & $\begin{array}{l}231 \\
(8.7)\end{array}$ & $236(9.2)$ & $\begin{array}{l}238 \\
(9.6)\end{array}$ & $166(6.8)$ & $112(4.3)$ \\
\hline Benin & $384(7.6)$ & 340 (7) & $490(9.9)$ & $\begin{array}{l}420 \\
(8.6)\end{array}$ & $\begin{array}{l}438 \\
(9.1)\end{array}$ & $\begin{array}{l}429 \\
(8.8)\end{array}$ & $\begin{array}{l}403 \\
(8.4)\end{array}$ & $\begin{array}{l}427 \\
(8.9)\end{array}$ & 432 (8.7) & 453 (9) & $328(6.8)$ & $353(7.2)$ \\
\hline Ivory C & $\begin{array}{l}208 \\
(10.2)\end{array}$ & 175 (8.8) & 149 (7.9) & $\begin{array}{l}192 \\
(10.3)\end{array}$ & $\begin{array}{l}174 \\
(8.2)\end{array}$ & $\begin{array}{l}174 \\
(8.2)\end{array}$ & $\begin{array}{l}168 \\
(7.4)\end{array}$ & $\begin{array}{l}145 \\
(8)\end{array}$ & $150(8.2)$ & $\begin{array}{l}166 \\
(9.2)\end{array}$ & $152(7)$ & 135 (6.5) \\
\hline Ghana & $130(7)$ & 115 (6.1) & $162(8.2)$ & $\begin{array}{l}165 \\
(9.8)\end{array}$ & $\begin{array}{l}174 \\
(9.6)\end{array}$ & $\begin{array}{l}191 \\
(11.2)\end{array}$ & $\begin{array}{l}163 \\
(9.5)\end{array}$ & $\begin{array}{l}151 \\
(8.4)\end{array}$ & $132(7.1)$ & $132(7)$ & 128 (7.7) & $135(8.4)$ \\
\hline Nigeria & $\begin{array}{l}1358 \\
(9.9)\end{array}$ & 1307 (9.5) & $1256(8.9)$ & $\begin{array}{l}1156 \\
(8.3)\end{array}$ & $\begin{array}{l}1089 \\
(7.6)\end{array}$ & $\begin{array}{l}1041 \\
(7.6)\end{array}$ & $\begin{array}{l}1080 \\
(7.6)\end{array}$ & $\begin{array}{l}1158 \\
(8.3)\end{array}$ & $1126(8.2)$ & $\begin{array}{l}1173 \\
(8.5)\end{array}$ & 1072 (7.6) & 1099 (8) \\
\hline
\end{tabular}

Sierra L : Sierra Leone. Ivory C : Ivory Coast. Countries are classified according to their Gross National Income (GNI) per capita (equivalent in US dollars).

Table 3: Comparison of the appearance of MOCS between countries by individual and household factors. 


\begin{tabular}{|c|c|c|c|c|c|c|c|c|c|c|c|c|}
\hline & Wealth & & & & & & Education & & & & & \\
\hline & Poor & & Middle & & Rich & & None & & Primary & & Secondary & \\
\hline & OR (Cl) & $\mathrm{P}$ & OR (Cl) & $\mathrm{P}$ & $\mathrm{OR}(\mathrm{Cl})$ & $\mathrm{P}$ & $\mathrm{OR}(\mathrm{Cl})$ & $\mathrm{P}$ & $\mathrm{OR}(\mathrm{Cl})$ & $\mathrm{P}$ & OR (Cl) & $\mathrm{P}$ \\
\hline Gambia & $\begin{array}{l}0.792(0.561- \\
1.118)\end{array}$ & & $\begin{array}{l}1.085(0.705- \\
1.669)\end{array}$ & & $\begin{array}{l}0.619(0.449- \\
0.853)\end{array}$ & & $\begin{array}{l}1.047(0.815- \\
1.344)\end{array}$ & & $\begin{array}{l}0.816(0.495- \\
1.346)\end{array}$ & & $\begin{array}{l}0.253(0.136- \\
0.473)\end{array}$ & \\
\hline Liberia & $\begin{array}{l}0.815(0.584- \\
1.137)\end{array}$ & & $\begin{array}{l}1.137(0.769- \\
1.68)\end{array}$ & & $\begin{array}{l}0.716(0.527- \\
0.971)\end{array}$ & & $\begin{array}{l}1.029(0.77- \\
1.375)\end{array}$ & & $\begin{array}{l}0.728(0.506- \\
1.047)\end{array}$ & & $\begin{array}{l}0.653(0.446- \\
0.957)\end{array}$ & \\
\hline $\begin{array}{l}\text { Sierra } \\
\text { Leone }\end{array}$ & $\begin{array}{l}0.62(0.458- \\
0.84)\end{array}$ & & $\begin{array}{l}0.667(0.457- \\
0.974)\end{array}$ & & $\begin{array}{l}0.939(0.741- \\
1.19)\end{array}$ & & $\begin{array}{l}0.914(0.747- \\
1.118)\end{array}$ & & $\begin{array}{l}0.256(0.135- \\
0.485)\end{array}$ & & $\begin{array}{l}0.765(0.524- \\
1.116)\end{array}$ & \\
\hline Togo & $\begin{array}{l}0.536(0.36- \\
0.799)\end{array}$ & & $\begin{array}{l}0.889(0.589- \\
1.343)\end{array}$ & & $\begin{array}{l}0.523(0.378- \\
0.725)\end{array}$ & & $\begin{array}{l}0.654(0.463- \\
0.923)\end{array}$ & & $\begin{array}{l}0.506(0.353- \\
0.726)\end{array}$ & & $\begin{array}{l}0.633(0.413- \\
0.969)\end{array}$ & \\
\hline Guinea & $\begin{array}{l}0.595(0.413- \\
0.858)\end{array}$ & & $\begin{array}{l}0.434(0.249- \\
0.76)\end{array}$ & & $\begin{array}{l}0.599(0.433- \\
0.828)\end{array}$ & & $\begin{array}{l}0.635(0.492- \\
0.82)\end{array}$ & & $\begin{array}{l}0.402(0.205- \\
0.787)\end{array}$ & 0.000 & $\begin{array}{l}0.449(0.215- \\
0.936)\end{array}$ & \\
\hline Niger & $\begin{array}{l}0.937(0.703- \\
1.25)\end{array}$ & & $\begin{array}{l}0.7(0.523- \\
1.134)\end{array}$ & 0.002 & $\begin{array}{l}1.063(0.854- \\
1.325)\end{array}$ & 0.000 & $\begin{array}{l}1.088(0.906- \\
1.305)\end{array}$ & 0.000 & $\begin{array}{l}0.75(0.456- \\
1.254)\end{array}$ & & $\begin{array}{l}0.682(0.312- \\
1.49)\end{array}$ & 0.0 \\
\hline Mali & $\begin{array}{l}0.785(0.581- \\
1.06)\end{array}$ & 0.000 & $\begin{array}{l}0.629(0.41- \\
0.964)\end{array}$ & & $\begin{array}{l}0.708(0.545- \\
0.92)\end{array}$ & & $\begin{array}{l}0.762(0.619- \\
0.939)\end{array}$ & & $\begin{array}{l}0.9(0.55- \\
1.473)\end{array}$ & & $\begin{array}{l}0.504(0.252- \\
1.008)\end{array}$ & \\
\hline Benin & $\begin{array}{l}1.361(1.116- \\
1.661)\end{array}$ & & $\begin{array}{l}1.244(0.958- \\
1.615)\end{array}$ & & $\begin{array}{l}1.75(1.503- \\
2.037)\end{array}$ & & $\begin{array}{l}1.544(1.333- \\
1.788)\end{array}$ & & $\begin{array}{l}1.485(1.169- \\
1.887)\end{array}$ & & $\begin{array}{l}1.918(1.504- \\
2.446)\end{array}$ & \\
\hline $\begin{array}{l}\text { Ivory } \\
\text { Coast }\end{array}$ & $\begin{array}{l}1.041(0.777- \\
1.394)\end{array}$ & & $\begin{array}{l}0.648(0.4- \\
1.051)\end{array}$ & & $\begin{array}{l}0.696(0.508- \\
0.954)\end{array}$ & & $\begin{array}{l}0.897(0.7- \\
1.148)\end{array}$ & & $\begin{array}{l}0.788(0.547- \\
1.136)\end{array}$ & & $\begin{array}{l}0.336(0.144- \\
0.787)\end{array}$ & \\
\hline Ghana & $\begin{array}{l}0.692(0.475- \\
1.009)\end{array}$ & & $\begin{array}{l}1.051(0.695- \\
1.589)\end{array}$ & & $\begin{array}{l}0.479(0.332- \\
0.693)\end{array}$ & & $\begin{array}{l}0.702(0.461- \\
1.069)\end{array}$ & & $\begin{array}{l}0.7(0.444- \\
1.102)\end{array}$ & & $\begin{array}{l}0.613(0.445- \\
0.845)\end{array}$ & \\
\hline \multirow[t]{3}{*}{ Nigeria } & Reference & & Reference & & Reference & & Reference & & Reference & & Reference & \\
\hline & Residence & & & & Mother age $\mathrm{g}$ & oup & & & & & & \\
\hline & urban & & rural & & $20-29$ years & & $30-39$ years & & $40-49$ years & & $15-19$ years & \\
\hline Gambia & $\begin{array}{l}0.646(0.484- \\
0.863)\end{array}$ & & $\begin{array}{l}0.832(0.62- \\
1.116)\end{array}$ & & $\begin{array}{l}0.986(0.62- \\
1.569)\end{array}$ & & $\begin{array}{l}1.15(0.772- \\
1.712)\end{array}$ & & $\begin{array}{l}1.404(0.71- \\
2.776)\end{array}$ & & $\begin{array}{l}2.628(0.426- \\
16.208)\end{array}$ & \\
\hline Liberia & $\begin{array}{l}0.706(0.546- \\
0.914)\end{array}$ & & $\begin{array}{l}0.88(0.653- \\
1.185)\end{array}$ & & $\begin{array}{l}1.202(0.792- \\
1.825)\end{array}$ & & $\begin{array}{l}0.863(0.599- \\
1.244)\end{array}$ & & $\begin{array}{l}0.847(0.434- \\
1.652)\end{array}$ & & $\begin{array}{l}2.098(0.338- \\
13.012)\end{array}$ & \\
\hline $\begin{array}{l}\text { Sierra } \\
\text { Leone }\end{array}$ & $\begin{array}{l}1.008(0.775- \\
1.31)\end{array}$ & & $\begin{array}{l}0.697(0.561- \\
0.866)\end{array}$ & & $\begin{array}{l}0.874(0.538- \\
1.419)\end{array}$ & & $\begin{array}{l}0.698(0.462- \\
1.054)\end{array}$ & & $\begin{array}{l}0.82(0.405- \\
1.658)\end{array}$ & & $\begin{array}{l}0.521(0.034- \\
8.065)\end{array}$ & \\
\hline Togo & $\begin{array}{l}0.488(0.346- \\
0.689)\end{array}$ & & $\begin{array}{l}0.709 \\
(0.539- \\
0.933)\end{array}$ & & $\begin{array}{l}0.711(0.426- \\
1.186)\end{array}$ & & $\begin{array}{l}0.677(0.439- \\
1.044)\end{array}$ & & $\begin{array}{l}0.87(0.426- \\
1.775)\end{array}$ & & $\begin{array}{l}2.206(0.358- \\
13.585)\end{array}$ & \\
\hline Guinea & $\begin{array}{l}0.546(0.371- \\
0.804)\end{array}$ & & $\begin{array}{l}0.611(0.465- \\
0.803)\end{array}$ & & $\begin{array}{l}1.359(0.898- \\
2.056)\end{array}$ & & $\begin{array}{l}1.298(0.913- \\
1.846)\end{array}$ & & $\begin{array}{l}1.237(0.654- \\
2.341)\end{array}$ & & $\begin{array}{l}0.941(0.118- \\
7.512)\end{array}$ & \\
\hline Niger & $\begin{array}{l}1.645(1.226- \\
2.207)\end{array}$ & 0.000 & $\begin{array}{l}0.961(0.793- \\
1.165)\end{array}$ & 0.000 & $\begin{array}{l}1.131(0.737- \\
1.735)\end{array}$ & 0.000 & $\begin{array}{l}0.9(0.618- \\
1.312)\end{array}$ & 0.000 & $\begin{array}{l}0.869(0.439- \\
1.718)\end{array}$ & 0.000 & $\begin{array}{l}0.513(0.052- \\
5.078)\end{array}$ & 0.6 \\
\hline Mali & $\begin{array}{l}0.885(0.645- \\
1.214)\end{array}$ & & $\begin{array}{l}0.739(0.594- \\
0.92)\end{array}$ & & $\begin{array}{l}2.037(1.414- \\
2.934)\end{array}$ & & $\begin{array}{l}2(1.468- \\
2.726)\end{array}$ & & $\begin{array}{l}2.13(1.202- \\
3.775)\end{array}$ & & $\begin{array}{l}2.021(0.313- \\
13.06)\end{array}$ & \\
\hline Benin & $\begin{array}{l}1.56(1.333- \\
1.825)\end{array}$ & & $\begin{array}{l}1.513(1.301- \\
1.761)\end{array}$ & & $\begin{array}{l}1.221(0.785- \\
1.9)\end{array}$ & & $\begin{array}{l}1.026(0.687- \\
1.531)\end{array}$ & & $\begin{array}{l}1.073(0.523- \\
2.203)\end{array}$ & & $\begin{array}{l}1.259(0.173- \\
9.181)\end{array}$ & \\
\hline $\begin{array}{l}\text { Ivory } \\
\text { Coast }\end{array}$ & $\begin{array}{l}0.721(0.537- \\
0.969)\end{array}$ & & $\begin{array}{l}0.865(0.667- \\
1.122)\end{array}$ & & $\begin{array}{l}1.048(0.64- \\
1.716)\end{array}$ & & $\begin{array}{l}0.761(0.506- \\
1.146)\end{array}$ & & $\begin{array}{l}0.684(0.32- \\
1.462)\end{array}$ & & NA & \\
\hline Ghana & $\begin{array}{l}0.552(0.404- \\
0.755)\end{array}$ & & $\begin{array}{l}0.742(0.543- \\
1.015)\end{array}$ & & $\begin{array}{l}1.394(0.983- \\
1.977)\end{array}$ & & $\begin{array}{l}1.211(0.9- \\
1.629)\end{array}$ & & $\begin{array}{l}1.357(0.794- \\
2.318)\end{array}$ & & $\begin{array}{l}1.392(0.246- \\
7.888)\end{array}$ & \\
\hline \multirow[t]{3}{*}{ Nigeria } & Reference & & Reference & & Reference & & Reference & & Reference & & Reference & \\
\hline & $\begin{array}{l}\text { Access to } \\
\text { information }\end{array}$ & & Source of water & & Sanitation & & Antenatal cares & & & & & \\
\hline & Yes & & Improved & & Improved & & None & & $\leq 3$ cares & & $\geq 4$ cares & \\
\hline Gambia & $\begin{array}{l}0.624(0.497- \\
0.784)\end{array}$ & & $\begin{array}{l}0.682(0.356- \\
1.307)\end{array}$ & & $\begin{array}{l}0.749(0.571- \\
0.983)\end{array}$ & & $\begin{array}{l}1.121(0.73- \\
1.722)\end{array}$ & & $\begin{array}{l}0.449(0.225- \\
0.899)\end{array}$ & & $\begin{array}{l}0.809(0.627- \\
1.045)\end{array}$ & \\
\hline Liberia & $\begin{array}{l}0.768(0.612- \\
0.962)\end{array}$ & & $\begin{array}{l}0.706(0.468- \\
1.064)\end{array}$ & & $\begin{array}{l}0.979(0.797- \\
1.203)\end{array}$ & & $\begin{array}{l}1.75(1.236- \\
2.478)\end{array}$ & & $\begin{array}{l}0.686(0.371- \\
1.266)\end{array}$ & & $\begin{array}{l}0.71(0.547- \\
0.921)\end{array}$ & \\
\hline $\begin{array}{l}\text { Sierra } \\
\text { Leone }\end{array}$ & $\begin{array}{l}0.767(0.626- \\
0.94)\end{array}$ & & $\begin{array}{l}0.624(0.465- \\
0.839)\end{array}$ & & $\begin{array}{l}0.82(0.685- \\
0.982)\end{array}$ & & $\begin{array}{l}0.791(0.588- \\
1.064)\end{array}$ & & $\begin{array}{l}0.568(0.29- \\
1.114)\end{array}$ & & $\begin{array}{l}0.798(0.643- \\
0.991)\end{array}$ & \\
\hline
\end{tabular}




\begin{tabular}{|c|c|c|c|c|c|c|c|c|c|c|c|c|}
\hline Togo & $\begin{array}{l}0.556(0.43- \\
0.719)\end{array}$ & & $\begin{array}{l}0.696(0.489- \\
0.992)\end{array}$ & & $\begin{array}{l}0.634(0.501- \\
0.803)\end{array}$ & & $\begin{array}{l}0.981(0.634- \\
1.517)\end{array}$ & & $\begin{array}{l}0.573(0.367- \\
0.894)\end{array}$ & & $\begin{array}{l}0.592(0.435 \\
0.805)\end{array}$ & \\
\hline Guinea & $\begin{array}{l}0.569(0.439- \\
0.736)\end{array}$ & & $\begin{array}{l}0.463(0.282- \\
0.758)\end{array}$ & & $\begin{array}{l}0.594(0.463- \\
0.763)\end{array}$ & & $\begin{array}{l}0.836(0.576- \\
1.214)\end{array}$ & & $\begin{array}{l}0.56(0.347- \\
0.906)\end{array}$ & & $\begin{array}{l}0.479(0.338- \\
0.678)\end{array}$ & \\
\hline Niger & $\begin{array}{l}0.939(0.775- \\
1.136)\end{array}$ & 0.000 & $\begin{array}{l}1.098(0.832- \\
1.451)\end{array}$ & 0.000 & $\begin{array}{l}1.013(0.85- \\
1.207)\end{array}$ & 0.000 & $\begin{array}{l}1.223(0.956- \\
1.565)\end{array}$ & 0.000 & $\begin{array}{l}1.041(0.76- \\
1.426)\end{array}$ & 0.000 & $\begin{array}{l}0.755(0.541 \\
1.054)\end{array}$ & 0.0 \\
\hline Mali & $\begin{array}{l}0.674(0.547- \\
0.83)\end{array}$ & & $\begin{array}{l}0.8(0.589- \\
1.088)\end{array}$ & & $\begin{array}{l}0.743(0.603- \\
0.916)\end{array}$ & & $\begin{array}{l}0.951(0.741- \\
1.221)\end{array}$ & & $\begin{array}{l}0.612(0.4- \\
0.937)\end{array}$ & & $\begin{array}{l}0.576(0.412- \\
0.806)\end{array}$ & \\
\hline Benin & $\begin{array}{l}1.546(1.364- \\
1.753)\end{array}$ & & $\begin{array}{l}1.825(1.463- \\
2.276)\end{array}$ & & $\begin{array}{l}1.589(1.402- \\
1.8)\end{array}$ & & $\begin{array}{l}1.737(1.448- \\
2.083)\end{array}$ & & $\begin{array}{l}1.209(0.884- \\
1.655)\end{array}$ & & $\begin{array}{l}1.696(1.452- \\
1.981)\end{array}$ & \\
\hline $\begin{array}{l}\text { Ivory } \\
\text { Coast }\end{array}$ & $\begin{array}{l}0.849(0.678- \\
1.062)\end{array}$ & & $\begin{array}{l}1.046(0.724- \\
1.512)\end{array}$ & & $\begin{array}{l}0.891(0.717- \\
1.107)\end{array}$ & & $\begin{array}{l}1.575(1.122- \\
2.211)\end{array}$ & & $\begin{array}{l}0.711(0.487- \\
1.037)\end{array}$ & & $\begin{array}{l}0.675(0.48- \\
0.949)\end{array}$ & \\
\hline Ghana & $\begin{array}{l}0.623(0.494- \\
0.785)\end{array}$ & & $\begin{array}{l}0.208(0.073- \\
0.591)\end{array}$ & & $\begin{array}{l}0.701(0.551- \\
0.891)\end{array}$ & & $\begin{array}{l}2.292(1.507- \\
3.486)\end{array}$ & & $\begin{array}{l}0.431(0.155- \\
1.197)\end{array}$ & & $\begin{array}{l}0.55(0.416- \\
0.727)\end{array}$ & \\
\hline Nigeria & Reference & & Reference & & Reference & & Reference & & Reference & & Reference & \\
\hline
\end{tabular}

OR: Odds Ratio from logistic regression models; Cl: Confidence Interval. NA: Not Applicable. Countries are classified according to their Gross National Income (GNI) per capita (equivalent in US dollars).

Table 3 Continued: 


\begin{tabular}{|c|c|c|c|c|c|c|c|c|c|c|c|c|}
\hline & \multicolumn{2}{|c|}{ Household members } & \multicolumn{4}{|c|}{ Child age group } & \multicolumn{2}{|l|}{ Gender } & \multicolumn{2}{|l|}{ Small birth size } & \multicolumn{2}{|l|}{ diarrhea } \\
\hline & $>5$ & & $<24$ months & & $\geq 24$ moths & & Male & & yes & & yes & \\
\hline & $\mathrm{OR}(\mathrm{Cl})$ & $\mathrm{P}$ & $\mathrm{OR}(\mathrm{Cl})$ & $\mathrm{P}$ & $\mathrm{OR}(\mathrm{Cl})$ & $\mathrm{P}$ & OR (Cl) & $P$ & $\mathrm{OR}(\mathrm{Cl})$ & $\mathrm{P}$ & $\mathrm{OR}(\mathrm{Cl})$ & $\mathrm{P}$ \\
\hline Gambia & $\begin{array}{l}0.683(0.548- \\
0.851)\end{array}$ & & $\begin{array}{l}0.993(0.774- \\
1.273)\end{array}$ & & $\begin{array}{l}0.993(0.774- \\
1.273)\end{array}$ & & $\begin{array}{l}0.745(0.561- \\
0.991)\end{array}$ & & $\begin{array}{l}0.884(0.668- \\
1.168)\end{array}$ & & $\begin{array}{l}0.888(0.566- \\
1.394)\end{array}$ & \\
\hline Liberia & $\begin{array}{l}0.79(0.619- \\
1.007)\end{array}$ & & $\begin{array}{l}0.9(0.708- \\
1.145)\end{array}$ & & $\begin{array}{l}0.9(0.708- \\
1.145)\end{array}$ & & $\begin{array}{l}0.909(0.705- \\
1.172)\end{array}$ & & $\begin{array}{l}0.815(0.61- \\
1.089)\end{array}$ & & $\begin{array}{l}0.954(0.64- \\
1.421)\end{array}$ & \\
\hline $\begin{array}{l}\text { Sierra } \\
\text { Leone }\end{array}$ & $\begin{array}{l}0.663(0.542- \\
0.812)\end{array}$ & & $\begin{array}{l}0.661(0.534- \\
0.819)\end{array}$ & & $\begin{array}{l}0.661(0.534- \\
0.819)\end{array}$ & & $\begin{array}{l}0.62(0.479- \\
0.803)\end{array}$ & & $\begin{array}{l}0.763(0.593- \\
0.983)\end{array}$ & & $\begin{array}{l}0.65(0.388- \\
1.09)\end{array}$ & \\
\hline Togo & $\begin{array}{l}0.597(0.455- \\
0.783)\end{array}$ & & $\begin{array}{l}0.655(0.505- \\
0.851)\end{array}$ & & $\begin{array}{l}0.655(0.505- \\
0.851)\end{array}$ & & $\begin{array}{l}0.596(0.441- \\
0.804)\end{array}$ & & $\begin{array}{l}0.514(0.347- \\
0.761)\end{array}$ & & $\begin{array}{l}0.441(0.238- \\
0.815)\end{array}$ & \\
\hline Guinea & $\begin{array}{l}0.54(0.422- \\
0.692)\end{array}$ & & $\begin{array}{l}0.64(0.488- \\
0.839)\end{array}$ & & $\begin{array}{l}0.64(0.488- \\
0.839)\end{array}$ & & $\begin{array}{l}0.629(0.471- \\
0.839)\end{array}$ & & $\begin{array}{l}0.678(0.501- \\
0.918)\end{array}$ & & $\begin{array}{l}0.432(0.239- \\
0.78)\end{array}$ & \\
\hline Niger & $\begin{array}{l}0.967(0.803- \\
1.165)\end{array}$ & 0.000 & $\begin{array}{l}0.976(0.795- \\
1.197)\end{array}$ & 0.000 & $\begin{array}{l}0.976(0.795- \\
1.197)\end{array}$ & 0.000 & $\begin{array}{l}1.062(0.856- \\
1.319)\end{array}$ & 0.000 & $\begin{array}{l}1.541(1.147- \\
2.071)\end{array}$ & 0.000 & $\begin{array}{l}1.086(0.745- \\
1.583)\end{array}$ & 0.0 \\
\hline Mali & $\begin{array}{l}0.693(0.561- \\
0.857)\end{array}$ & & $\begin{array}{l}0.779(0.63- \\
0.962)\end{array}$ & & $\begin{array}{l}0.779(0.63- \\
0.962)\end{array}$ & & $\begin{array}{l}0.597(0.456- \\
0.781)\end{array}$ & & $\begin{array}{l}0.723(0.55- \\
0.951)\end{array}$ & & $\begin{array}{l}0.448(0.216- \\
0.929)\end{array}$ & \\
\hline Benin & $\begin{array}{l}1.53(1.336- \\
1.753)\end{array}$ & & $\begin{array}{l}1.402(1.217- \\
1.615)\end{array}$ & & $\begin{array}{l}1.402(1.217- \\
1.615)\end{array}$ & & $\begin{array}{l}1.652(1.421- \\
1.919)\end{array}$ & & $\begin{array}{l}1.666(1.33- \\
2.087)\end{array}$ & & $\begin{array}{l}1.575(1.085- \\
2.286)\end{array}$ & \\
\hline $\begin{array}{l}\text { Ivory } \\
\text { Coast }\end{array}$ & $\begin{array}{l}0.822(0.653- \\
1.033)\end{array}$ & & $\begin{array}{l}0.917(0.718- \\
1.172)\end{array}$ & & $\begin{array}{l}0.917(0.718- \\
1.172)\end{array}$ & & $\begin{array}{l}0.904(0.691- \\
1.182)\end{array}$ & & $\begin{array}{l}0.646(0.472- \\
0.882)\end{array}$ & & $\begin{array}{l}0.62(0.378- \\
1.018)\end{array}$ & \\
\hline Ghana & $\begin{array}{l}0.766(0.569- \\
1.031)\end{array}$ & & $\begin{array}{l}0.777(0.597- \\
1.011)\end{array}$ & & $\begin{array}{l}0.777(0.597- \\
1.011)\end{array}$ & & $\begin{array}{l}0.741(0.555- \\
0.989)\end{array}$ & & $\begin{array}{l}0.725(0.534- \\
0.985)\end{array}$ & & $\begin{array}{l}0.645(0.337- \\
1.235)\end{array}$ & \\
\hline \multirow[t]{3}{*}{ Nigeria } & Reference & & Reference & & Reference & & Reference & & Reference & & Reference & \\
\hline & \multicolumn{12}{|l|}{ Month of birth } \\
\hline & January & & February & & March & & April & & May & & June & \\
\hline Gambia & $\begin{array}{l}1.111 \\
(0.639- \\
1.931)\end{array}$ & & $\begin{array}{l}0.43(0.19- \\
0.973)\end{array}$ & & $\begin{array}{l}1.019(0.54- \\
1.926)\end{array}$ & & $\begin{array}{l}1.334 \\
(0.684- \\
2.602)\end{array}$ & & $\begin{array}{l}0.884 \\
(0.419- \\
1.865)\end{array}$ & & $\begin{array}{l}1.06(0.533- \\
2.109)\end{array}$ & \\
\hline Liberia & $\begin{array}{l}0.422 \\
(0.174- \\
1.025)\end{array}$ & & $\begin{array}{l}0.495 \\
(0.222- \\
1.103)\end{array}$ & & $\begin{array}{l}0.564 \\
(0.265- \\
1.198)\end{array}$ & & $\begin{array}{l}1.572 \\
(0.899- \\
2.748)\end{array}$ & & $\begin{array}{l}0.903 \\
(0.495- \\
1.645)\end{array}$ & & $\begin{array}{l}0.99(0.544- \\
1.802)\end{array}$ & \\
\hline $\begin{array}{l}\text { Sierra } \\
\text { Leone }\end{array}$ & $\begin{array}{l}0.499 \\
(0.256- \\
0.974)\end{array}$ & & $\begin{array}{l}0.736 \\
(0.428- \\
1.267)\end{array}$ & & $\begin{array}{l}0.677 \\
(0.404- \\
1.133)\end{array}$ & & $\begin{array}{l}0.66(0.381- \\
1.146)\end{array}$ & & $\begin{array}{l}0.454(0.24- \\
0.858)\end{array}$ & & $\begin{array}{l}0.854 \\
(0.512- \\
1.424)\end{array}$ & \\
\hline Togo & $\begin{array}{l}1.228 \\
(0.652- \\
2.312)\end{array}$ & & $\begin{array}{l}0.344 \\
(0.131- \\
0.902)\end{array}$ & & $\begin{array}{l}0.731(0.39- \\
1.371)\end{array}$ & & $\begin{array}{l}0.977 \\
(0.529- \\
1.803)\end{array}$ & & $\begin{array}{l}0.478 \\
(0.236- \\
0.967)\end{array}$ & & $\begin{array}{l}0.761 \\
(0.392- \\
1.478)\end{array}$ & \\
\hline Guinea & $\begin{array}{l}0.332 \\
(0.128- \\
0.861)\end{array}$ & 0.000 & $\begin{array}{l}0.527 \\
(0.279- \\
0.995)\end{array}$ & 0.000 & $\begin{array}{l}0.735 \\
(0.416- \\
1.299)\end{array}$ & 0.172 & $\begin{array}{l}0.828 \\
(0.439- \\
1.561)\end{array}$ & 0.003 & $\begin{array}{l}0.503 \\
(0.246- \\
1.027)\end{array}$ & 0.000 & $\begin{array}{l}0.394 \\
(0.179- \\
0.869)\end{array}$ & 0.0 \\
\hline Niger & $\begin{array}{l}0.74(0.432- \\
1.267)\end{array}$ & & $\begin{array}{l}0.634 \\
(0.377- \\
1.068)\end{array}$ & & $\begin{array}{l}1.075 \\
(0.693- \\
1.668)\end{array}$ & & $\begin{array}{l}0.878 \\
(0.492- \\
1.565)\end{array}$ & & $\begin{array}{l}1.171 \\
(0.709- \\
1.933)\end{array}$ & & $\begin{array}{l}1.367 \\
(0.817- \\
2.287)\end{array}$ & \\
\hline Mali & $\begin{array}{l}0.4(0.169- \\
0.95)\end{array}$ & & $\begin{array}{l}0.615 \\
(0.322- \\
1.174)\end{array}$ & & $\begin{array}{l}1.033 \\
(0.639-1.67)\end{array}$ & & $\begin{array}{l}1.125(0.66- \\
1.916)\end{array}$ & & $\begin{array}{l}0.642 \\
(0.362- \\
1.139)\end{array}$ & & $\begin{array}{l}0.648 \\
(0.358- \\
1.173)\end{array}$ & \\
\hline Benin & $\begin{array}{l}1.997 \\
(1.393- \\
2.864)\end{array}$ & & $\begin{array}{l}1.778(1.25- \\
2.53)\end{array}$ & & $\begin{array}{l}1.31(0.931- \\
1.843)\end{array}$ & & $\begin{array}{l}1.916 \\
(1.325- \\
2.771)\end{array}$ & & $\begin{array}{l}1.45(0.997- \\
2.107)\end{array}$ & & $\begin{array}{l}1.36(0.927- \\
1.995)\end{array}$ & \\
\hline $\begin{array}{l}\text { Ivory } \\
\text { Coast }\end{array}$ & $\begin{array}{l}1.418 \\
(0.855- \\
2.354)\end{array}$ & & $\begin{array}{l}0.7(0.371- \\
1.321)\end{array}$ & & $\begin{array}{l}0.531(0.25- \\
1.127)\end{array}$ & & $\begin{array}{l}0.499 \\
(0.228- \\
1.091)\end{array}$ & & $\begin{array}{l}0.301 \\
(0.109- \\
0.835)\end{array}$ & & $\begin{array}{l}0.704 \\
(0.347- \\
1.428)\end{array}$ & \\
\hline Ghana & $\begin{array}{l}1.243 \\
(0.643- \\
2.402)\end{array}$ & & $\begin{array}{l}0.819 \\
(0.389- \\
1.724)\end{array}$ & & $\begin{array}{l}1.148 \\
(0.646-2.04)\end{array}$ & & $\begin{array}{l}1.141 \\
(0.624- \\
2.089)\end{array}$ & & $\begin{array}{l}0.359 \\
(0.143- \\
0.903)\end{array}$ & & $\begin{array}{l}0.496 \\
(0.235- \\
1.044)\end{array}$ & \\
\hline \multirow[t]{3}{*}{ Nigeria } & Reference & & Reference & & Reference & & Reference & & Reference & & Reference & \\
\hline & \multicolumn{12}{|l|}{ Month of birth } \\
\hline & July & & August & & September & & October & & November & & December & \\
\hline
\end{tabular}




\begin{tabular}{|c|c|c|c|c|c|c|c|c|c|c|c|c|}
\hline Gambia & $\begin{array}{l}0.448(0.163- \\
1.229)\end{array}$ & & $\begin{array}{l}0.682(0.316- \\
1.474)\end{array}$ & & $\begin{array}{l}0.745(0.394- \\
1.41)\end{array}$ & & $\begin{array}{l}0.289(0.102- \\
0.816)\end{array}$ & & $\begin{array}{l}0.773(0.371- \\
1.609)\end{array}$ & & $\begin{array}{l}0.79(0.377- \\
1.653)\end{array}$ & \\
\hline Liberia & $\begin{array}{l}0.662(0.335- \\
1.306)\end{array}$ & & $\begin{array}{l}1.68(0.944- \\
2.991)\end{array}$ & & $\begin{array}{l}0.918(0.499- \\
1.691)\end{array}$ & & $\begin{array}{l}0.86(0.423- \\
1.745)\end{array}$ & & $\begin{array}{l}0.628(0.279- \\
1.414)\end{array}$ & & $\begin{array}{l}0.561(0.215 \\
1.469)\end{array}$ & \\
\hline $\begin{array}{l}\text { Sierra } \\
\text { Leone }\end{array}$ & $\begin{array}{l}0.592(0.326- \\
1.076)\end{array}$ & & $\begin{array}{l}0.818(0.437- \\
1.53)\end{array}$ & & $\begin{array}{l}1.085(0.634- \\
1.855)\end{array}$ & & $\begin{array}{l}0.823(0.436- \\
1.553)\end{array}$ & & $\begin{array}{l}1.606(0.926- \\
2.786)\end{array}$ & & $\begin{array}{l}0.858(0.422 \\
1.746)\end{array}$ & \\
\hline Togo & $\begin{array}{l}0.7(0.345- \\
1.418)\end{array}$ & & $\begin{array}{l}0.336(0.125- \\
0.906)\end{array}$ & & $\begin{array}{l}0.261(0.104- \\
0.659)\end{array}$ & & $\begin{array}{l}0.565(0.266- \\
1.202)\end{array}$ & & $\begin{array}{l}0.224(0.059- \\
0.844)\end{array}$ & & $\begin{array}{l}1.109(0.519- \\
2.371)\end{array}$ & \\
\hline Guinea & $\begin{array}{l}0.526(0.242- \\
1.141)\end{array}$ & 0.005 & $\begin{array}{l}0.871(0.407- \\
1.863)\end{array}$ & 0.001 & $\begin{array}{l}0.596(0.281- \\
1.266)\end{array}$ & 0.027 & $\begin{array}{l}0.207(0.038- \\
1.143)\end{array}$ & 0.000 & $\begin{array}{l}0.659(0.27- \\
1.609)\end{array}$ & 0.005 & $\begin{array}{l}0.244(0.052 \\
1.137)\end{array}$ & 0.2 \\
\hline Niger & $\begin{array}{l}0.821(0.431- \\
1.563)\end{array}$ & & $\begin{array}{l}0.921(0.51- \\
1.664)\end{array}$ & & $\begin{array}{l}0.788(0.446- \\
1.392)\end{array}$ & & $\begin{array}{l}1.411(0.832- \\
2.392)\end{array}$ & & $\begin{array}{l}1.224(0.631- \\
2.372)\end{array}$ & & $\begin{array}{l}1.164(0.594 \\
2.278)\end{array}$ & \\
\hline Mali & $\begin{array}{l}0.722(0.396- \\
1.317)\end{array}$ & & $\begin{array}{l}1.108(0.647- \\
1.899)\end{array}$ & & $\begin{array}{l}0.389(0.191- \\
0.793)\end{array}$ & & $\begin{array}{l}0.42(0.2- \\
0.88)\end{array}$ & & $\begin{array}{l}0.668(0.31- \\
1.442)\end{array}$ & & $\begin{array}{l}1.109(0.478 \\
2.572)\end{array}$ & \\
\hline Benin & $\begin{array}{l}1.571(1.089- \\
2.268)\end{array}$ & & $\begin{array}{l}1.769(1.223- \\
2.559)\end{array}$ & & $\begin{array}{l}0.934(0.624- \\
1.398)\end{array}$ & & $\begin{array}{l}1.774(1.238- \\
2.542)\end{array}$ & & $\begin{array}{l}1.544(0.988- \\
2.412)\end{array}$ & & $\begin{array}{l}1.603(1.007 \\
2.554)\end{array}$ & \\
\hline $\begin{array}{l}\text { Ivory } \\
\text { Coast }\end{array}$ & $\begin{array}{l}1.094(0.593- \\
2.018)\end{array}$ & & $\begin{array}{l}1.707(0.991- \\
2.94)\end{array}$ & & $\begin{array}{l}1.387(0.822- \\
2.34)\end{array}$ & & $\begin{array}{l}0.174(0.048- \\
0.628)\end{array}$ & & $\begin{array}{l}0.427(0.15- \\
1.22)\end{array}$ & & $\begin{array}{l}0.834(0.342 \\
2.033)\end{array}$ & \\
\hline Ghana & $\begin{array}{l}0.5(0.229- \\
1.09)\end{array}$ & & $\begin{array}{l}0.313(0.104- \\
0.941)\end{array}$ & & $\begin{array}{l}0.667(0.309- \\
1.441)\end{array}$ & & $\begin{array}{l}0.575(0.234- \\
1.411)\end{array}$ & & $\begin{array}{l}0.226(0.055- \\
0.93)\end{array}$ & & $\begin{array}{l}0.668(0.267 \\
1.671)\end{array}$ & \\
\hline Nigeria & Reference & & Reference & & Reference & & Reference & & Reference & & Reference & \\
\hline
\end{tabular}

OR: Odds Ratio from logistic regression models; Cl: Confidence Interval. Countries are classified according to their Gross National Income (GNI) per capita (equivalent in US dollars).

Table 4: Multivariate logistic regression of the association of each factors with the MOCS by country 


\begin{tabular}{|c|c|c|c|c|c|c|}
\hline & GAMBIA & LIBERIA & SIERRAL & TOGO & GUINEA & NIGER \\
\hline & OR $(95 \% \mathrm{Cl})$ & OR $(95 \% \mathrm{Cl})$ & OR $(95 \% \mathrm{Cl})$ & OR $(95 \% \mathrm{Cl})$ & OR $(95 \% \mathrm{Cl})$ & OR $(95 \% \mathrm{Cl})$ \\
\hline Place of residence (Rural) & $\begin{array}{l}1.346(0.724- \\
2.505)\end{array}$ & $\begin{array}{l}1.004(0.594- \\
1.698)\end{array}$ & $\begin{array}{l}1.548(1.004- \\
2.387)\end{array}$ & $\begin{array}{l}0.556(0.256- \\
1.206)\end{array}$ & $\begin{array}{l}0.923(0.475- \\
1.793)\end{array}$ & $\begin{array}{l}2.545 * *(1.644- \\
3.94)\end{array}$ \\
\hline \multicolumn{7}{|l|}{ Wealth (Poor) } \\
\hline Middle & $\begin{array}{l}1.621(0.911- \\
2.884)\end{array}$ & $\begin{array}{l}1.932 * \\
3.409)\end{array}$ & $\begin{array}{l}1.382(0.867- \\
2.203)\end{array}$ & $\begin{array}{l}3.082 \text { ** (1.706- } \\
5.568)\end{array}$ & $\begin{array}{l}0.929(0.473- \\
1.823)\end{array}$ & $\begin{array}{l}1.221(0.766- \\
1.946)\end{array}$ \\
\hline Rich & $\begin{array}{l}1.345(0.666- \\
2.716)\end{array}$ & $\begin{array}{l}2.136^{\star}(1.137- \\
4.011)\end{array}$ & $\begin{array}{l}2.082^{\star}(1.283- \\
3.379)\end{array}$ & $\begin{array}{l}3.809 * *(1.513- \\
9.589)\end{array}$ & $\begin{array}{l}1.968(0.991- \\
3.907)\end{array}$ & $\begin{array}{l}1.651 *(1.079- \\
2.525)\end{array}$ \\
\hline \multicolumn{7}{|l|}{ Mother age (20-29-year) } \\
\hline 30-39-year & $\begin{array}{l}1.395(0.871- \\
2.235)\end{array}$ & $\begin{array}{l}2.215^{\star \star}(1.395- \\
3.517)\end{array}$ & $\begin{array}{l}1.303(0.905- \\
1.875)\end{array}$ & $\begin{array}{l}1.317(0.801- \\
2.166)\end{array}$ & $\begin{array}{l}1.629(0.958- \\
2.77)\end{array}$ & $\begin{array}{l}1.801 * *(1.269- \\
2.555)\end{array}$ \\
\hline 40-49-year & $\begin{array}{l}1.346(0.687- \\
2.635)\end{array}$ & $\begin{array}{l}2.97 \star \star \\
5.416)\end{array}$ & $\begin{array}{l}1.296(0.767- \\
2.191)\end{array}$ & $\begin{array}{l}1.686(0.876- \\
3.247)\end{array}$ & $\begin{array}{l}2.062^{*}(1.06- \\
4.014)\end{array}$ & $\begin{array}{l}1.687 *(1.031- \\
2.76)\end{array}$ \\
\hline 15-19-year & $\begin{array}{l}0.444(0.078- \\
2.516)\end{array}$ & $\begin{array}{l}1.222(0.526- \\
2.836)\end{array}$ & $\begin{array}{l}0.698(0.309- \\
1.577)\end{array}$ & $\begin{array}{l}0.274(0.03- \\
2.526)\end{array}$ & $\begin{array}{l}1.671(0.71- \\
3.935)\end{array}$ & $\begin{array}{l}0.354(0.099- \\
1.269)\end{array}$ \\
\hline \multicolumn{7}{|l|}{ Education ( $\geq$ Secondary) } \\
\hline None & $\begin{array}{l}3.737 * *(1.833- \\
7.618)\end{array}$ & $\begin{array}{l}1.351(0.779- \\
2.342)\end{array}$ & $\begin{array}{l}1.54(0.964- \\
2.462)\end{array}$ & $\begin{array}{l}0.916(0.476- \\
1.762)\end{array}$ & $\begin{array}{l}1.36(0.585- \\
3.163)\end{array}$ & $\begin{array}{l}2.23(0.948- \\
5.247)\end{array}$ \\
\hline Primary & $\begin{array}{l}4.473^{\star *}(1.936- \\
10.332)\end{array}$ & $\begin{array}{l}1.36(0.778- \\
2.377)\end{array}$ & $\begin{array}{l}0.513(0.243- \\
1.08)\end{array}$ & $\begin{array}{l}0.906(0.504- \\
1.629)\end{array}$ & $\begin{array}{l}1.007(0.364- \\
2.788)\end{array}$ & $1.564(0.6-4.077)$ \\
\hline $\begin{array}{l}\text { Access to information } \\
\text { (No) }\end{array}$ & $\begin{array}{l}0.505^{\star}(0.281- \\
0.907)\end{array}$ & $\begin{array}{l}1.068(0.672- \\
1.696)\end{array}$ & $\begin{array}{l}1.164(0.817- \\
1.659)\end{array}$ & $\begin{array}{l}0.95(0.573- \\
1.574)\end{array}$ & $\begin{array}{l}1.402(0.821- \\
2.394)\end{array}$ & $\begin{array}{l}0.942(0.669- \\
1.326)\end{array}$ \\
\hline $\begin{array}{l}\text { Source of water } \\
\text { (Unimproved) }\end{array}$ & $\begin{array}{l}0.795(0.385- \\
1.641)\end{array}$ & $\begin{array}{l}0.673(0.411- \\
1.101)\end{array}$ & $\begin{array}{l}0.79(0.541- \\
1.153)\end{array}$ & $\begin{array}{l}1.043(0.627- \\
1.734)\end{array}$ & $\begin{array}{l}0.742(0.412- \\
1.335)\end{array}$ & $1.333(0.935-1.9)$ \\
\hline $\begin{array}{l}\text { Sanitation Facility } \\
\text { (Unimproved) }\end{array}$ & $0.809(0.516-1.27)$ & $\begin{array}{l}2.815^{\star}(1.223- \\
6.48)\end{array}$ & $\begin{array}{l}1.259(0.708- \\
2.239)\end{array}$ & $\begin{array}{l}0.79(0.404- \\
1.547)\end{array}$ & $\begin{array}{l}0.979(0.54- \\
1.772)\end{array}$ & $\begin{array}{l}0.93(0.566- \\
1.527)\end{array}$ \\
\hline Household Members (<5) & $1.216(0.583-2.54)$ & $\begin{array}{l}1.061(0.707- \\
1.592)\end{array}$ & $\begin{array}{l}1.008(0.711- \\
1.43)\end{array}$ & $\begin{array}{l}1.446(0.904- \\
2.312)\end{array}$ & $\begin{array}{l}1.34(0.754- \\
2.383)\end{array}$ & $\begin{array}{l}1.075(0.742- \\
1.556)\end{array}$ \\
\hline \multicolumn{7}{|l|}{ Antenatal care ( $>4$ Times) } \\
\hline None & $\begin{array}{l}0.768(0.439- \\
1.343)\end{array}$ & $\begin{array}{l}2.626 * *(1.649- \\
4.181)\end{array}$ & $\begin{array}{l}1.181(0.819- \\
1.702)\end{array}$ & $\begin{array}{l}2.04^{*}(1.122- \\
3.711)\end{array}$ & $2^{\star}(1.145-3.494)$ & 2 ** $(1.263-3.166)$ \\
\hline$<3$ Times & $\begin{array}{l}0.587(0.283- \\
1.215)\end{array}$ & $\begin{array}{l}1.102 \text { (0.573- } \\
2.119)\end{array}$ & $\begin{array}{l}0.713(0.36- \\
1.413)\end{array}$ & $\begin{array}{l}1.114(0.662- \\
1.875)\end{array}$ & $\begin{array}{l}1.467(0.82- \\
2.624)\end{array}$ & $\begin{array}{l}1.412(0.936- \\
2.13)\end{array}$ \\
\hline Child age (>24 month) & $\begin{array}{l}4.038^{\star \star}(2.457- \\
6.637)\end{array}$ & $\begin{array}{l}1.858 * * \\
2.869)\end{array}$ & $\begin{array}{l}1.215(0.867- \\
1.703)\end{array}$ & $\begin{array}{l}2.474 * \star \\
3.978)\end{array}$ & $\begin{array}{l}2.424^{\star *}(1.486- \\
3.953)\end{array}$ & $\begin{array}{l}1.526^{*}(1.068- \\
2.181)\end{array}$ \\
\hline Child Gender (female) & $\begin{array}{l}1.073(0.705- \\
1.634)\end{array}$ & $\begin{array}{l}0.749(0.503- \\
1.114)\end{array}$ & $\begin{array}{l}1.42^{\star}(1.028- \\
1.962)\end{array}$ & $\begin{array}{l}0.906(0.591- \\
1.387)\end{array}$ & $\begin{array}{l}0.75(0.478- \\
1.177)\end{array}$ & $\begin{array}{l}0.825(0.609- \\
1.116)\end{array}$ \\
\hline Small Birth Weight (Yes) & $\begin{array}{l}1.252(0.817- \\
1.917)\end{array}$ & $\begin{array}{l}0.792 \text { (0.533- } \\
1.177)\end{array}$ & $\begin{array}{l}1.008(0.731- \\
1.39)\end{array}$ & $\begin{array}{l}0.774(0.485- \\
1.235)\end{array}$ & $\begin{array}{l}1.245(0.797- \\
1.944)\end{array}$ & $\begin{array}{l}1.498^{*}(1.054- \\
2.131)\end{array}$ \\
\hline Child Diarrhea (No) & $\begin{array}{l}1.299(0.776- \\
2.173)\end{array}$ & $\begin{array}{l}1.232(0.787- \\
1.93)\end{array}$ & $\begin{array}{l}0.772 \text { (0.458- } \\
1.3)\end{array}$ & $\begin{array}{l}0.745(0.391- \\
1.419)\end{array}$ & $\begin{array}{l}0.719(0.384- \\
1.347)\end{array}$ & $\begin{array}{l}1.234(0.835- \\
1.824)\end{array}$ \\
\hline \multicolumn{7}{|l|}{$\begin{array}{l}\text { Month of birth } \\
\text { (December) }\end{array}$} \\
\hline January & $\begin{array}{l}2.093(0.841- \\
5.206)\end{array}$ & $\begin{array}{l}1.072(0.294- \\
3.912)\end{array}$ & $\begin{array}{l}0.966(0.381- \\
2.452)\end{array}$ & $\begin{array}{l}1.546(0.589- \\
4.059)\end{array}$ & $\begin{array}{l}2.241(0.371- \\
13.545)\end{array}$ & $\begin{array}{l}1.028(0.453- \\
2.335)\end{array}$ \\
\hline February & $\begin{array}{l}0.882(0.293- \\
2.655)\end{array}$ & $\begin{array}{l}1.513(0.438- \\
5.224)\end{array}$ & $\begin{array}{l}1.636(0.699- \\
3.831)\end{array}$ & $\begin{array}{l}0.557(0.166- \\
1.872)\end{array}$ & $\begin{array}{l}3.754(0.721- \\
19.551)\end{array}$ & $\begin{array}{l}1.211(0.537- \\
2.733)\end{array}$ \\
\hline March & $2.166(0.82-5.719)$ & $\begin{array}{l}1.799(0.537- \\
6.02)\end{array}$ & $\begin{array}{l}1.502(0.654- \\
3.45)\end{array}$ & $\begin{array}{l}1.402(0.537- \\
3.664)\end{array}$ & $\begin{array}{l}5.434^{*}(1.064- \\
27.749)\end{array}$ & $\begin{array}{l}1.735(0.812- \\
3.707)\end{array}$ \\
\hline April & $2.103(0.79-5.593)$ & $\begin{array}{l}3.318^{*}(1.113- \\
9.89)\end{array}$ & $\begin{array}{l}1.066(0.459- \\
2.479)\end{array}$ & $\begin{array}{l}1.095(0.425- \\
2.821)\end{array}$ & $\begin{array}{l}5.328(1.025- \\
27.693)\end{array}$ & $\begin{array}{l}1.101(0.474- \\
2.56)\end{array}$ \\
\hline May & $\begin{array}{l}1.691(0.588- \\
4.859)\end{array}$ & $\begin{array}{l}2.447 \text { (0.808- } \\
7.408)\end{array}$ & $\begin{array}{l}0.877(0.355- \\
2.168)\end{array}$ & $\begin{array}{l}0.621(0.228- \\
1.693)\end{array}$ & $\begin{array}{l}3.528(0.657- \\
18.959)\end{array}$ & $\begin{array}{l}1.522(0.69- \\
3.353)\end{array}$ \\
\hline June & $\begin{array}{l}1.591(0.573- \\
4.421)\end{array}$ & $\begin{array}{l}2.709 \text { (0.896- } \\
8.186)\end{array}$ & $\begin{array}{l}1.734(0.763- \\
3.938)\end{array}$ & $\begin{array}{l}0.964(0.364- \\
2.558)\end{array}$ & $\begin{array}{l}2.772 \text { (0.497- } \\
15.45)\end{array}$ & $\begin{array}{l}1.903(0.855- \\
4.237)\end{array}$ \\
\hline July & $1.034(0.298-$ & $1.63(0.509-$ & $1.161(0.483-$ & $1.038(0.379-$ & 3.974 (0.718- & $1.345(0.551-$ \\
\hline
\end{tabular}

Page 12/19 


\begin{tabular}{|c|c|c|c|c|c|c|}
\hline & $3.585)$ & $5.219)$ & 2.791) & $2.843)$ & 22.015) & $3.287)$ \\
\hline August & $\begin{array}{l}1.008(0.353- \\
2.878)\end{array}$ & $\begin{array}{l}3.495^{\star}(1.158- \\
10.55)\end{array}$ & $\begin{array}{l}1.371(0.559- \\
3.363)\end{array}$ & $\begin{array}{l}0.378(0.111- \\
1.29)\end{array}$ & $\begin{array}{l}5.261(0.956- \\
28.97)\end{array}$ & $\begin{array}{l}1.196(0.51- \\
2.805)\end{array}$ \\
\hline September & $\begin{array}{l}1.654(0.635- \\
4.309)\end{array}$ & $\begin{array}{l}3.426 *(1.116- \\
10.521)\end{array}$ & $\begin{array}{l}2.358(1.014- \\
5.483)\end{array}$ & $\begin{array}{l}0.38(0.117- \\
1.229)\end{array}$ & $\begin{array}{l}4.995(0.908- \\
27.483)\end{array}$ & $\begin{array}{l}1.265(0.546- \\
2.934)\end{array}$ \\
\hline October & $\begin{array}{l}0.513(0.145- \\
1.815)\end{array}$ & $2.382(0.73-7.78)$ & $\begin{array}{l}1.347(0.546- \\
3.324)\end{array}$ & $\begin{array}{l}0.736(0.26- \\
2.084)\end{array}$ & $\begin{array}{l}1.708(0.172- \\
16.98)\end{array}$ & $\begin{array}{l}1.908(0.843- \\
4.315)\end{array}$ \\
\hline November & $\begin{array}{l}1.438(0.518- \\
3.993)\end{array}$ & $\begin{array}{l}1.557(0.452- \\
5.359)\end{array}$ & $\begin{array}{l}2.441 *(1.056- \\
5.644)\end{array}$ & $\begin{array}{l}0.244(0.054- \\
1.098)\end{array}$ & $\begin{array}{l}3.655(0.629- \\
21.253)\end{array}$ & $\begin{array}{l}1.446(0.586- \\
3.566)\end{array}$ \\
\hline
\end{tabular}

OR: Odds Ratio from logistic regression models; Cl: Confidence Interval. ${ }^{\star} \mathrm{P}<0.05 ;{ }^{*} \mathrm{P}<0.01$. NA: Not Applicable.

Table 4 continued: 


\begin{tabular}{|c|c|c|c|c|c|}
\hline & MALI & BENIN & IVORY COAST & GHANA & NIGERIA \\
\hline & OR $(95 \% \mathrm{Cl})$ & OR $(95 \% \mathrm{Cl})$ & OR $(95 \% \mathrm{Cl})$ & OR $(95 \% \mathrm{Cl})$ & OR $(95 \% \mathrm{Cl})$ \\
\hline Place of residence (Rural) & $1.906 *(1.129-3.217)$ & $1.185(0.949-1.478)$ & $2.425^{\star}(1.188-4.948)$ & $1.165(0.637-2.131)$ & $1.248^{\star \star}(1.059-1.47)$ \\
\hline \multicolumn{6}{|l|}{ Wealth (Poor) } \\
\hline Middle & $1.003(0.601-1.673)$ & $1.214(0.913-1.614)$ & $0.546(0.269-1.108)$ & $2.092 *(1.064-4.115)$ & $\begin{array}{l}1.345^{\star \star}(1.105- \\
1.636)\end{array}$ \\
\hline Rich & $1.357(0.779-2.363)$ & $\begin{array}{l}2.009 * *(1.533- \\
2.634)\end{array}$ & $0.605(0.264-1.387)$ & $1.259(0.548-2.894)$ & $\begin{array}{l}1.694 \star \star \\
2.119)\end{array}$ \\
\hline \multicolumn{6}{|l|}{ Mother age (20-29-year) } \\
\hline 30-39-year & $1.593 *(1.062-2.389)$ & $\begin{array}{l}1.844 \star \star \\
2.273)\end{array}$ & $1.489(0.957-2.315)$ & $1.176(0.692-1.997)$ & $\begin{array}{l}1.448 \star \star \\
1.679)\end{array}$ \\
\hline 40-49-year & $1.397(0.795-2.457)$ & $\begin{array}{l}2.239 * \star(1.641- \\
3.053)\end{array}$ & $1.564(0.831-2.943)$ & $1.08(0.512-2.281)$ & $\begin{array}{l}1.629 * \star(1.339- \\
1.982)\end{array}$ \\
\hline 15-19-year & $0.213^{*}(0.042-1.064)$ & $0.437(0.185-1.031)$ & $0.432(0.136-1.367)$ & \#V (LUE! & $\begin{array}{l}0.461^{\star \star}(0.282- \\
0.756)\end{array}$ \\
\hline \multicolumn{6}{|l|}{ Education ( $\geq$ Secondary) } \\
\hline None & $1.182(0.539-2.59)$ & $1.028(0.766-1.381)$ & $2.911 *(1.152-7.356)$ & $0.927(0.483-1.781)$ & $1.22 *(1.006-1.479)$ \\
\hline Primary & $1.565(0.651-3.76)$ & $1.018(0.744-1.393)$ & $3.259 *(1.26-8.431)$ & $1.203(0.658-2.199)$ & $1.252^{*}(1.044-1.502)$ \\
\hline Access to information (No) & $0.945(0.623-1.435)$ & $1.129(0.896-1.424)$ & $\begin{array}{l}2.335^{\star \star}(1.403- \\
3.885)\end{array}$ & $1.762(0.709-4.375)$ & $1.208 *(1.023-1.426)$ \\
\hline Source of water (Unimproved) & $1.024(0.679-1.546)$ & $1.145(0.91-1.441)$ & $1.382(0.864-2.21)$ & $\begin{array}{l}0.191 * \star \\
0.597)\end{array}$ & $0.997(0.855-1.162)$ \\
\hline $\begin{array}{l}\text { Sanitation Facility } \\
\text { (Unimproved) }\end{array}$ & $0.885(0.573-1.366)$ & $0.912(0.695-1.197)$ & $1.233(0.681-2.235)$ & $0.762(0.373-1.555)$ & $0.855^{\star}(0.746-0.98)$ \\
\hline Household Members (<5) & $1.105(0.728-1.678)$ & $1.217(0.994-1.489)$ & $1.66 *(1.055-2.61)$ & $\begin{array}{l}1.873^{\star \star}(1.148- \\
3.056)\end{array}$ & $\begin{array}{l}1.248 * \star \\
1.445)\end{array}$ \\
\hline \multicolumn{6}{|l|}{ Antenatal care (>4 Times) } \\
\hline None & $\begin{array}{l}2.134 \star *(1.352- \\
3.368)\end{array}$ & $1.268^{\star}(1.012-1.59)$ & $\begin{array}{l}2.264 \star \star \\
3.934)\end{array}$ & $\begin{array}{l}4.936^{\star \star}(2.701- \\
9.019)\end{array}$ & $\begin{array}{l}1.348 * \star(1.158- \\
1.568)\end{array}$ \\
\hline$<3$ Times & $1.317(0.789-2.2)$ & $0.785(0.597-1.033)$ & $1.035(0.628-1.704)$ & $1.062(0.358-3.149)$ & $1.168(0.919-1.483)$ \\
\hline Child age (>24 month) & $2.59 * \star(1.721-3.898)$ & $1.266 *(1.036-1.546)$ & $\begin{array}{l}1.842^{\star \star}(1.201- \\
2.827)\end{array}$ & $\begin{array}{l}2.406^{\star *}(1.442- \\
4.015)\end{array}$ & $\begin{array}{l}1.654 * *(1.446- \\
1.893)\end{array}$ \\
\hline Child Gender (female) & $1.466 *(1.032-2.083)$ & $0.884(0.736-1.062)$ & $0.808(0.543-1.202)$ & $0.824(0.517-1.314)$ & $0.977(0.861-1.108)$ \\
\hline Small Birth Weight (Yes) & $0.982(0.689-1.399)$ & $0.987(0.781-1.247)$ & $\begin{array}{l}0.577 * \star \\
0.868)\end{array}$ & $1.023(0.649-1.614)$ & $0.894(0.786-1.016)$ \\
\hline Child Diarrhea (No) & $0.759(0.359-1.604)$ & $0.974(0.689-1.376)$ & $0.59(0.347-1.003)$ & $0.899(0.438-1.845)$ & $1.128(0.922-1.381)$ \\
\hline \multicolumn{6}{|l|}{ Month of birth (December) } \\
\hline January & $0.482(0.148-1.574)$ & $1.93^{\star \star}(1.172-3.179)$ & $2.116(0.771-5.813)$ & $1.735(0.554-5.44)$ & $1.466 *(1.051-2.046)$ \\
\hline February & $0.997(0.354-2.806)$ & $\begin{array}{l}2.073^{\star \star}(1.258- \\
3.414)\end{array}$ & $1.496(0.506-4.424)$ & $1.653(0.501-5.452)$ & $\begin{array}{l}1.821 * \star \\
2.519)\end{array}$ \\
\hline March & $1.861(0.731-4.741)$ & $1.397(0.859-2.271)$ & $0.88(0.274-2.829)$ & $2.436(0.828-7.169)$ & $\begin{array}{l}1.767^{\star *}(1.272- \\
2.454)\end{array}$ \\
\hline April & $1.46(0.561-3.801)$ & $1.613(0.984-2.644)$ & $0.722(0.225-2.319)$ & $1.921(0.649-5.689)$ & $1.335(0.942-1.891)$ \\
\hline May & $1.024(0.385-2.719)$ & $1.306(0.793-2.149)$ & $0.526(0.139-1.998)$ & $0.628(0.173-2.279)$ & $1.506 *(1.065-2.129)$ \\
\hline June & $1.039(0.385-2.803)$ & $1.299(0.784-2.154)$ & $1.331(0.434-4.085)$ & $0.846(0.26-2.746)$ & $1.541^{\star \star}(1.09-2.179)$ \\
\hline July & $1.27(0.467-3.452)$ & $1.607(0.978-2.638)$ & $2.416(0.829-7.045)$ & $0.959(0.284-3.235)$ & $1.69 * \star(1.199-2.381)$ \\
\hline August & $1.337(0.511-3.495)$ & $1.61(0.982-2.64)$ & $2.405(0.866-6.683)$ & $0.448(0.106-1.902)$ & $1.374(0.971-1.946)$ \\
\hline September & $0.627(0.215-1.828)$ & $1.022(0.602-1.735)$ & $3.137 *(1.139-8.642)$ & $1.528(0.457-5.107)$ & $1.788^{\star \star}(1.28-2.499)$ \\
\hline October & $0.497(0.168-1.47)$ & $1.764^{*}(1.081-2.878)$ & $0.285(0.06-1.349)$ & $0.852(0.232-3.135)$ & $1.477 *(1.048-2.081)$ \\
\hline November & $0.837(0.279-2.513)$ & $1.176(0.681-2.029)$ & $0.55(0.141-2.137)$ & $0.303(0.056-1.644)$ & $1.274(0.886-1.83)$ \\
\hline
\end{tabular}

OR: Odds Ratio from logistic regression models; Cl: Confidence Interval. *P < 0.05; ** P< 0.01. NA: Not Applicable. 
Table 5: Comparison of the prevalence of stunting, overweight and MOSC between countries.

\begin{tabular}{|lllllll|}
\hline & STUNTING & & OVERWEIGHT & & MOCS \\
\hline Country(rank*) & OR $(95 \% \mathrm{Cl})$ & P-value & OR $(95 \% \mathrm{Cl})$ & P-value & OR (95\%Cl) & P-value \\
\hline Gambia(1) & 1 (reference) & & 1 (reference) & & 1 (reference) & \\
\hline Liberia(2) & $1.245(1.082-1.431)$ & 0.002 & $1.136(0.974-1.325)$ & 0.105 & $1.091(0.833-1.428)$ & 0.526 \\
\hline Sierra Leone(3) & $1.756(1.548-1.992)$ & 0.000 & $0.654(0.563-0.760)$ & 0.000 & $0.992(0.773-1.274)$ & 0.950 \\
\hline TOGO(4) & $1.067(0.928-1.227)$ & 0.360 & $1.332(1.147-1.546)$ & 0.000 & $0.798(0.600-1.060)$ & 0.119 \\
\hline Guinea(5) & $1.335(1.164-1.532)$ & 0.000 & $0.732(0.623-0.861)$ & 0.000 & $0.738(0.553-0.987)$ & 0.040 \\
\hline Niger(6) & $2.736(2.407-3.110)$ & 0.000 & $0.779(0.670-0.905)$ & 0.001 & $1.295(1.014-1.654)$ & 0.038 \\
\hline Mali(7) & $2.012(1.769-2.288)$ & 0.000 & $0.696(0.597-0.811)$ & 0.000 & $0.951(0.735-1.231)$ & 0.704 \\
\hline Benin(8) & $2.369(2.108-2.663)$ & 0.000 & $1.216(1.068-1.385)$ & 0.003 & $2.049(1.651-2.542)$ & 0.000 \\
\hline Ivory Coast(9) & $1.232(1.072-1.416)$ & 0.003 & $1.013(0.867-1.183)$ & 0.872 & $1.054(0.805-1.381)$ & 0.701 \\
\hline Ghana(10) & $0.645(0.552-0.752)$ & 0.000 & $2.372(2.046-2.750)$ & 0.000 & $0.873(0.654-1.165)$ & 0.357 \\
\hline Nigeria(11) & $1.829(1.643-2.037)$ & 0.000 & $1.159(1.030-1.305)$ & 0.015 & $1.317(1.072-1.618)$ & 0.009 \\
\hline
\end{tabular}

* Ranked from the lowest to the highest Gross National Income; Source: World Bank 2015 Country Data and Statistics website, accessed July 2020.

Table 5 shows a comparison of the prevalence of stunting, overweight and MOSC between countries ranked by Gross national income. Taking the country with the lowest GNI 2015 that Gambia as the reference country. The proportion of stunting was significantly high in all countries except Togo and Ghana, this proportion was two times higher in Niger, Mali and Benin [odds ratio (95\% Cl): 2.736 (2.407-3.11), 2.012 (1.769-2.288), 2.369 (2.108-2.663), respectively]. The proportion of mother overweight was significantly low in 4 countries (Sierra Leone, , Guinea, Niger and Mali), significantly high in 4 countries (Togo, Benin, Ghana and Nigeria) and non-significantly high in 2 countries (Liberia Ivory Coast). The proportion of MOSC was significantly different compare to Gambia in Guinea, Niger, Benin and Nigeria [odds ratio (95\%Cl): 0.738 (0.553-0.987), 1.295 (1.014-1.654), 2.049 (1.651-2.542), 1.317 (1.072-1.618), respectively], while there was marginal or no difference compare to Gambia in the other 6 countries.

\section{Discussion}

The patterns of associations between the individual or household factors and MOSC occurrence varied widely across the country data sets examined and was independently linked to their gross national income per capita.

In our study, the proportion of MOSC was more likely to occur in the urban area than the rural area in most West African countries. One explanation may be that the nutritional transition and sedentary lifestyles may more favor MOSC occurrence in urban areas. An analysis on pooled data of sub-Saharan Africa from a recent study showed a strong association between living in urban and MOSC occurrence[13], another study in which African countries are included reported that the association of mother overweight and stunting children with urbanization do not differ between rural and urban areas [11]. In Bangladesh, DBM was more likely to be more prevalent in urban areas [19], and authors state that the double burden is more likely to occur in rural areas in many LMICs [1, 12].

Rich or middle households' wealth index were more associated with higher odds of MOSC occurrence compared to poor households' wealth index in all west African countries except Ivory Coast. Even if a significant association was found in 5 countries, MOSC was more likely to appear in the wealthier household but this occurrence was non-linked to the level of a country's GNI. One study reported that MOSC is associated with higher levels of a country's GDP per capita but that it is perhaps the poorest households in those countries that tend to have the MOSC [11]. A study in Guatemala showed that the coexistence of female obesity and stunting children was significantly associated with the middle economic quintile [20]. Other studies report that the prevalence of DBM seems to increase with an increase in the wealth index of households $[1,12,18,19]$.

Mother age group was significantly associated with the MOSC occurrence in 5 countries Liberia, Niger, Benin, Mali and Nigeria. MOSC was more likely to occur in the 30-39- and 40-49-year age groups than in 20-29-year, this can be explained by the fact that the increase in a woman's age is associated with the overweight occurrence.

In Bangladesh, the maternal age group of 35 to 49 years was associated with the risk of the coexistence overweight/obese mother and child under-nutrition [19] 
Children over 24 months of age were associated with higher odds of MOSC occurrence compared to under 24 months in all countries except Sierra Leone. This may be explained by the fact of the gradual accumulation of episodes of malnutrition during the first years of childhood thus resulting in chronic conditions in advanced age[21]. The same result has been founded in pooled analysis on 14 African countries [13].In Bangladesh, the 24 to 49-month age group was associated with a high risk of the coexistence overweight/obese mother and child under-nutrition [19].

Mother with primary and/or secondary education were associated with higher odds of MOSC occurrence compared to those with no education in all countries except Togo and Guinea, however, MOSC was more likely to appear in mother with primary education with a significant association of more than three times in Mali and Gambia, and more than two times in Ivory Coast, while secondary education was significantly associated Ivory Coast (with more than three times), Gambia (with more than four times) and Nigeria. Other study found that Higher education in mother was associated with lower odds of MOSC, but was not among the factors strongly associated with it [13].

An explanation can be the cultural aspect that could interact in the sense that obesity considers as a mark of respect and admiration by society [8, 22], educated people who move or are already in urban areas are more attached to tradition and growth fat voluntarily. Studies highlighted the pressure of the tradition on mother lead them to growth fat [23].

Mother's lack of access to information was significantly associated with lower odds of MOSC occurrence in the Gambia, while the reverse is observed in Ivory Coast and Nigeria in which it was significantly associated with higher odds of MOSC occurrence. This can be explained by the fact that most of the population are living in rural areas, have low education and access to information is limited [21].

Household access to the unimproved water source was only significantly associated with lower odds of occurrence of MOSC in Ghana, other countries do not show a significant association. Household access to unimproved sanitation was significantly associated with MOSC occurrence in two countries which are Liberia and Nigeria, while diarrhea in children was marginal or not associated with the occurrence of MOSC in all countries.

Diarrheal disease and infection are the most common cause of unimproved water supply and sanitation thus leading to stunting children. A recent study found that Improved sanitation was significantly associated with MOSC in sub-Saharan Africa [13]. However, in this study, it was households with an improved water source that were more likely to have a high proportion of MOSC in Ghana. This can be explained by inadequate conservation or storage of drinking water in households. Cultural practice, the availability of safe water and accessibility to soap can be an obstacle to good hygiene practice for the control of infection prevention in households [24]. Households made up of more than 5 members was significantly associated with the MOSC occurrence in the 3 countries with the highest gross income per capita which are Ivory Coast, Ghana and Nigeria. A study in Mali in 2006 reported that household size was not associated with the prevalence of stunting or overweight but the high number of household members is a protective factor against the prevalence of stunting [8].

Antenatal cares were significantly associated with MOSC occurrence in 7 countries: Liberia, Niger, Benin, Mali, Ivory Coast, Ghana and Nigeria, women who did not have antenatal cares were more likely to be exposed to the MOSC occurrence than those who had more than 4 antenatal care. That same association was observed in Guinea although antenatal cares didn't show stronger significance. The MOSC occurrence was not linked to the GNI of the country, for example, the MOSC was 4 times more likely to occur among women who did not have antenatal cares in Ghana while it was less than 2 times in Nigeria. This can be explained by the easy access to health care and the quality of the consultations in each country, this can be explained by the easy access to health care and the quality of the consultations in each country. The authors of a study from three Latin American countries found that the association of the prenatal consultation with stunting differs between countries and that is due to the quality of each country's prenatal consultation [25]. Another study in Nigeria concluded that a poor prenatal visit is associated with a risk of poor nutritional status in young children [26].

Child sex was not significantly associated with MOSC in the majority of countries except Sierra Leone and Mali, where MOSC was more likely to appear significantly in female than in the male. Studies in Nigeria and Bangladesh reported that malnutrition was more associated with female gender, while that association was strong among male gender in South Africa. Others studies in Brazil shown than male gender was associated with MOSC occurrence compared to female gender [26-29].

Children small birth weight was only significantly associated with the MOSC occurrence that in two countries, in Niger, it was those who had small birth weight who seemed to be more affected by the MOSC while the opposite was observed in Ivory Coast where small birth weight appeared the most affected. Studies in Bangladesh and Indonesia have reported a positive association between low birth weight and stunting children [30] [31]. An explanation may be that countries, in which normal or higher birth weight was more associated with the MOSC occurrence may have uncontrolled access of individuals or households to adequate, sufficient and necessary food for an active and healthy life[32]. Another explanation can be the change in individual and family eating habits, a welleducated people living in urban areas may not have acquired food knowledge to feed properly their children, and for those living in rural areas will always face food insecurity from the area whatever the wealth index levels. Studies reported that the available food resources especially exported food may not be used adequately by many mother $[8,33,34]$, leading to malnutrition and even after controlling by socio-economic factors, food insecurity in an area has a huge influence on nutritional status $[8,19]$.

Children month of birth were significantly associated with the MOSC occurrence in the four highest GNI per capita in 2015 countries: Benin, Ivory Coast, Ghana and Nigeria. In Ivory Coast, September was significantly associated with higher odds of MOSC occurrence compared to December, in Ghana no month compared to December showed significant association but the month of birth covariate was significantly associated with the MOSC occurrence, whereas in Nigeria all months were significantly associated with higher odds of MOSC occurrence compared to December except April and August. In Liberia, April, August and September were both significantly associated with higher odds of MOSC occurrence compared to December, but the covariate did not show statistical significance on analysis. 
A study on the effect of month of birth on children's health reported that the association between the month of birth and stunting children depended on the geographic position of countries. The authors have reported that this association decreases after the child's second year of life[35]. In India, the month of birth was significantly associated with stunting [36].

A comparison between countries showed that the MOSC occurrence was not necessarily related to countries' GNI and it was difficult to say that it was related to the proportion of stunting or that of overweight. Taking the country with the lowest GNI as a reference, the prevalence of stunting was significantly higher in all countries except Togo in which it was not significantly high and Ghana in which stunting was rather significantly low compared to that of Gambia. Whereas the overweight prevalence was two times as high in Ghana but the MOSC did not show a significant difference compared to the Gambia. The prevalence of stunting was two times higher in Mali, Niger and Benin than other countries, while overweight in Niger was significantly low and that of Benin was significantly high but these two countries showed a significantly high prevalence of MOSC compared to The Gambia. We can believe that the prevalence of MOSC does not seem to be linked to the high prevalence of stunting or overweight but more to the high prevalence of both in the same country. The point we can make is that the MOSC in West African countries does not show such a large difference in proportion, not in the same way as that of overweight or stunting which shows large differences between countries.

Taking as a reference the previous studies on African countries the prevalence of MOSC has increased, for example, it increased from $2.2 \%$ in 1993 to $5.3 \%$ in 2014 in Ghana, from 2.0\% in 1996 to 11.52\% in 2013 in Benin. Apart from Benin all the other countries have their prevalence of MOSC below 10\%.

\section{Conclusion}

In this study, none of the individual and household factors were strongly associated in the same way with MOSC occurrence in West African countries, they varied considerably between national databases and independent of national income per capita. However, Children's age and antenatal cares were the two covariates strongly associated with MOSC occurrence in most countries (10 and 7 out of 11 countries, respectively). The prevalence of MOSC does not seem to be linked to the high prevalence of stunting or overweight but more to the high prevalence of both in the same country. The message for decision-makers would be to establish specific measures according to the factors associated with the MOSC of each country and not measures common to all countries.

\section{List Of Abbreviations}

LMICs: low- and middle-income countries

MOSC: coexistence of mother overweight and stunting children in the same household

OR: Odds Ratios

Cl: Confidence Interval

DHS: Demographic and Health Survey

BMl: body mass index

HAZ: height for age z-scores

GNI: Gross National Income

\section{Declarations}

Ethics approval and consent to participate : Ethical approval to conduct the DHS survey was obtained from the National Ethics Committee for Health and Life Sciences (CNESS) of the Ministry of Health and Public Hygiene and the Ethics Committee ( Institutional Review Board) of ICF.

Consent for publication: Not applicable.

Availability of data and materials: The datasets analysed during the current study are available

from the DHS website (https://dhsprogram.com/).

Competing interests: The authors (SST, QL, ANA, HZ and YH) declare that they have no competing interests.

Funding: The author(s) disclosed receipt of the following financial support for the research, authorship, and/or publication of this article: This work was supported by the National Natural Science Foundation of China [Project number 81673165].

Authors' contributions: the study was designed by SST and QL; SST performed the data collection, extraction and analysis, under the guidance of ANA, HZ and $\mathrm{YH}$. All authors contributed to the verification and interpretation of the results and QL revised the final draft manuscript. All authors have read and approved the final version of the manuscript.

Acknowledgments The authors are grateful to the Zhengzhou University and its College of Public Health for providing an environment that promotes research and the National Natural Science Foundation of China for its support.

Authors' information (optional)

Page 17/19 
Stanislav Seydou Traore: PhD student in Nutrition and food hygiene department

Adwoa Nyantakyiwaa Amoah: PhD student in Nutrition and food hygiene department

Han Zhang: Master's student in Nutrition and food hygiene department

Youchun Hu:, Master's student in Nutrition and food hygiene department

Quanjun Lyu : Professor in charge of all those students listed above.

\section{References}

1. Popkin BM, Corvalan C, Grummer-Strawn LM. Dynamics of the double burden of malnutrition and the changing nutrition reality. Lancet. 2020;395(10217):65-74. Doi:10.1016/S0140-6736(19)32497-3

2. Development initiatives, 2018. 2018 global nutrition report: Shining a light to spur action on nutrition. Bristol, uk: Development initiatives.

3. Cigar. Time to act on malnutrition in all its forms in west africa by roos verstraeten. March 2, 2020. Https://a4nh.Cgiar.Org/2020/03/02/time-to-act-onmalnutrition-in-all-its-forms-in-west-africa/. Accessed [4 june 2020].

4. Onyango AW, Jean-Baptiste J, Samburu B, Mahlangu TLM. Regional overview on the double burden of malnutrition and examples of program and policy responses: African region. Ann Nutr Metab. 2019;75(2):127-30. Doi:10.1159/000503671

5. World health organization. Obesity and overweight[[updated 1 April 2020https://www.who.int/news-room/fact-sheets/detail/obesity-and-overweight. [accessed 4 june2020]

6. Unicef/ world health organization/world bank. Joint child malnutrition estimates, edition[2018 [updated september 2019http://www.who.int/nutgrowthdb/estimates2017/en/.[accessed 4 june 2020]

7. Global nutrition report. Mali nutrition profile[[updated 2019https://globalnutritionreport.org/resources/nutrition-profiles/africa/western-africa/mali/. [accessed 4 june2020]

8. Samba diop et al, la montée de l'obésité dans un contexte où la dénutrition n'est pas éradiquée. Application de l'approche des capabilités sur des données mixtes au mali. . African Population Studies. 2014:Vol 28, No. 02.

9. World health organisation.Malnutrition[2020.[updated 2020.https://www.who.int/health-topics/malnutrition\#tab=tab_1.[accessed 19 aug2020]

10. World health organization. Nutrition. Stunting in a nutshell[2020.[https://www.who.int/nutrition/healthygrowthproj_stunted_videos/en/.[19 August 2020]

11. Garrett JL, Ruel MT. Stunted child-overweight mother pairs: Prevalence and association with economic development and urbanization. Food Nutr Bull. 2005;26(2):209-21. Doi:10.1177/156482650502600205

12. Garrett J, Ruel MT. The coexistence of child undernutrition and maternal overweight: Prevalence, hypotheses, and programme and policy implications. Matern Child Nutr. 2005;1(3):185-96. Doi:10.1111/j.1740-8709.2005.00034.x

13. Acharya y, naz s, galway Ip and jones ad (2020) deforestation and household- and individual-level double burden of malnutrition in sub-saharan africa. Front Sustain Food Syst 4:33. Doi:doi: 10.3389/fsufs.2020.00033

14. Wells JC, et al. The double burden of malnutrition: Aetiological pathways and consequences for health. Lancet. 2020;395(10217):75-88 Doi:10.1016/S0140-6736(19)32472-9

15. Hoffman D. The double burden of malnutrition: Research agenda for reversing global trends. Ann Nutr Metab. 2019;75(2):149-52. Doi:10.1159/000503676

16. Institut national de la statistique (instat), cellule de planification et de statistique secteur santé-développement social et promotion de la famille (cps/ssds-pf) et icf. 2019. Enquête démographique et de santé au mali 2018. Bamako, mali et rockville, maryland, USA : Instat, cps/ss-ds-pf et icf.

17. Croft, trevor n., aileen m. J. Marshall, courtney k. Allen, et al.Guide to dhs statistics. . Rockville, Maryland, USA: ICF. 2018.

18. Dembélé B. Coexistence du surpoids ou obésité et retard de croissance dans les ménages du sud-ouest bénin. Santé Publique. 2018;1 (Vol. 30), p. 115124. Doi:10.3917/spub.181.0115

19. Hauqe SE, Sakisaka K, Rahman M. Examining the relationship between socioeconomic status and the double burden of maternal over and child undernutrition in bangladesh. Eur J Clin Nutr. 2019;73(4):531-40. Doi:10.1038/s41430-018-0162-6

20. Lee J, Houser RF, Must A, de Fulladolsa PP, Bermudez OI. Socioeconomic disparities and the familial coexistence of child stunting and maternal overweight in guatemala. Econ Hum Biol. 2012;10(3):232-41. Doi:10.1016/j.ehb.2011.08.002

21. Morrisson, c. Et c. Linskens (2000), « les facteurs explicatifs de la malnutrition en afrique subsaharienne », documents de travail du centre de développement de l'ocde, n 167, éditions ocde, paris. Doi:https://doi.org/10.1787/513743835456

22. Grijalva-Eternod CS, et al. The double burden of obesity and malnutrition in a protracted emergency setting: A cross-sectional study of western sahara refugees. PLoS Med. 2012;9(10):e1001320. Doi:10.1371/journal.pmed.1001320

23. Rguibi M, Belahsen R. Fattening practices among moroccan saharawi women. East Mediterr Health J. 2006;12(5):619-24.

24. Who. Global nutrition targets 2025: Stunting policy brief (who/nmh/nhd/14.3). Geneva: World health organization; 2014.

25. Forero-Ramirez N, Gamboa LF, Bedi A, Sparrow R. Child malnutrition and prenatal care: Evidence from three latin american countries. Rev Panam Salud Publica. 2014;35(3):163-71. 
26. Hamel $\mathrm{C}$, et al. Childhood malnutrition is associated with maternal care during pregnancy and childbirth: A cross-sectional study in bauchi and cross river states, nigeria. J Public Health Res. 2015;4(1):408. Doi:10.4081/jphr.2015.408

27. Gubert MB, Spaniol AM, Segall-Correa AM, Perez-Escamilla R. Understanding the double burden of malnutrition in food insecure households in brazil. Matern Child Nutr. 2017;13(3). Doi:10.1111/mcn.12347

28. Choudhury KK, Hanifı MA, Rasheed S, Bhuiya A. Gender inequality and severe malnutrition among children in a remote rural area of bangladesh. J Health Popul Nutr. 2000;18(3):123-30.

29. Lesiapeto $\mathrm{ms}$, smuts $\mathrm{cm}$, hanekom sm, et al. Risk factors of poor anthropometric status in children under five years of age living in rural districts of the eastern cape of kwazulu-natal provinces, south africa. S afr j clin nutr 2010;23:202-7.

30. Aryastami, n.K., shankar, a., kusumawardani, n. Et al. Low birth weight was the most dominant predictor associated with stunting among children aged 12-23 months in indonesia. Bmc nutr 3, 16 (2017). . Doi:https://doi.org/10.1186/s40795-017-0130-x

31. Rahman MS, Howlader T, Masud MS, Rahman ML. Association of low-birth weight with malnutrition in children under five years in bangladesh: Do mother's education, socio-economic status, and birth interval matter? PLoS One. 2016;11(6):e0157814. Doi:10.1371/journal.pone.0157814

32. Mother and child nutrition; mother, infant and young child nutrition and malnutrition[[updated 14 September, 2019 https://motherchildnutrition.org/malnutrition/about-malnutrition/underlying-causes-of-malnutrition.html [accessed 4 june 2020]

33. Popkin BM, Adair LS, Ng SW. Global nutrition transition and the pandemic of obesity in developing countries. Nutr Rev. 2012;70(1):3-21. Doi:10.1111/j.1753-4887.2011.00456.x

34. Popkin B. Global nutrition dynamics: The world is shifting rapidly toward a diet linked with noncommunicable diseases. Am J Clin Nutr. 2006;84:289-98.

35. Dorelien AM. Effects of birth month on child health and survival in sub-saharan africa. Biodemography Soc Biol. 2015;61(2):209-30.

Doi:10.1080/19485565.2015.1032399

36. Lokshin m, radyakin s. Month of birth and children's health in india. Journal of human resources. 2012; 47:174-203. Doi:10.3368/jhr.47.1.174

\section{Figures}

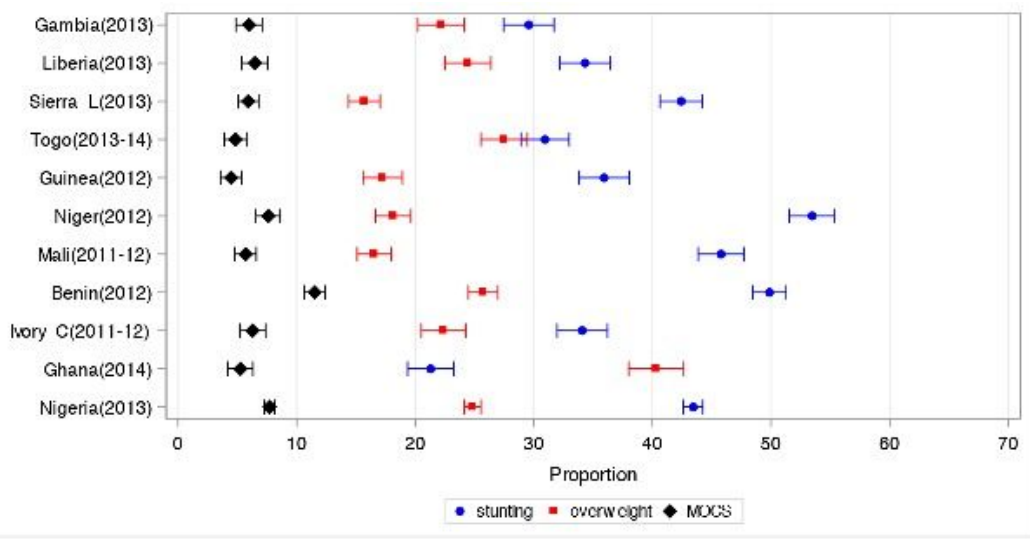

\section{Figure 1}

Prevalence of women overweight, stunting children and MOCS. Sierra_L : Sierra Leone. Ivory_C : Ivory Coast. Countries are ranked by their Gross National Income per capita (equivalent in US Dollars).

\section{Supplementary Files}

This is a list of supplementary files associated with this preprint. Click to download.

- Additionalfile1.docx 